\title{
Chemical amplification of magnetic field effects relevant to avian magnetoreception
}

\author{
Daniel R. Kattnig*1, Emrys W. Evans*², Victoire Déjean ${ }^{2}$, Charlotte A. Dodson ${ }^{3,4}$, \\ Mark I. Wallace ${ }^{3}$, Stuart R. Mackenzie ${ }^{1}$, Christiane R. Timmel ${ }^{2}$ and P. J. Hore ${ }^{1}$ \\ *These authors contributed equally. \\ ${ }^{1}$ Department of Chemistry, University of Oxford, Physical \& Theoretical Chemistry \\ Laboratory, Oxford, UK \\ ${ }^{2}$ Department of Chemistry, University of Oxford, Inorganic Chemistry Laboratory, \\ Oxford, UK \\ ${ }^{3}$ Department of Chemistry, University of Oxford, Chemistry Research Laboratory, \\ Oxford, UK \\ ${ }^{4}$ Current address: Molecular Medicine, National Heart \& Lung Institute, Imperial \\ College London, UK
}

\begin{abstract}
Magnetic fields as weak as the Earth's can change the yields of radical pair reactions even though the energies involved are orders of magnitude smaller than $k_{\mathrm{B}} T$ at room temperature. Proposed as the source of the light-dependent magnetic compass in migratory birds, the radical pair mechanism is thought to operate in cryptochrome flavoproteins in the retina. Here we demonstrate that the primary magnetic field effect on flavin photoreactions can be chemically amplified by slow radical termination reactions under conditions of continuous photoexcitation. The nature and origin of the amplification are revealed by studies of the intermolecular flavin-tryptophan and flavin-ascorbic acid photocycles and the closely related intramolecular flavin-tryptophan radical pair in cryptochrome. Amplification factors of up to 5.6 have been observed for magnetic fields weaker than $1 \mathrm{mT}$. Substantial chemical amplification could have a significant impact on the viability of a cryptochrome-based magnetic compass sensor.
\end{abstract}


Amongst other directional cues, migratory birds use a light-dependent geomagnetic compass for orientation and navigation ${ }^{1-3}$. Although the primary detection mechanism is unclear, the evidence currently points to magnetically sensitive photochemical reactions in cryptochrome proteins located in the retina ${ }^{4-}$ ${ }^{6}$. Cryptochromes ${ }^{7}$ contain the chromophore flavin adenine dinucleotide (FAD), photoexcitation of which can trigger three consecutive intra-protein electron transfers along a conserved triad of tryptophan (Trp) residues to produce a $\left(F A D^{\circ-} \operatorname{Trp}^{\bullet+}\right.$ ) radical pair ${ }^{8-10}$. This form of cryptochrome is magnetically sensitive in vitro and possibly also in vivo ${ }^{11-13}$. Similar magnetosensitivity is conceivable in other cryptochrome-derived radical pairs in which $\mathrm{FAD}^{\bullet-}$ or its protonated form, $\mathrm{FADH}^{\circ}$, is paired with an ascorbic acid radical ${ }^{14}$ or, less plausibly, superoxide, $\mathrm{O}_{2}^{\circ-}$ ${ }^{15,16}$. There appear to be different electron transfer pathways in some cryptochromes ${ }^{17,18}$, but no evidence so far that they give rise to magnetic field effects.

The radical pair mechanism is well established as the source of magnetic field effects on chemical reactions ${ }^{19}$. Remarkably, the magnetic interactions of electron spins in organic radicals can result in significant changes in reaction kinetics and product yields even though those interactions are many orders of magnitude weaker than the thermal energy, $k_{\mathrm{B}} T$. The sensitivity to applied magnetic fields derives from the coherent spin dynamics of pairs of radicals formed in spincorrelated states. Several conditions need to be satisfied for a radical pair to be suitable as a geomagnetic compass sensor ${ }^{5}$ : (a) the electron spin in at least one of the radicals must interact anisotropically with nuclear spins; (b) the mutual interaction of the two electron spins should be small; (c) the radical pair must react spin-selectively; (d) its lifetime should not be too short; and (e) the electron spin relaxation must be relatively slow. Studies of isolated cryptochrome in vitro suggest that the $\left(F A D^{--} \operatorname{Trp}^{\circ+}\right)$ radical pair is fit for the purpose of magnetic sensing ${ }^{11}$ although effects of Earth-strength magnetic fields and directional responses have yet to be demonstrated.

Evidence that cryptochrome plays a sensory role in magnetoreception is slowly accumulating but still largely circumstantial $\left.\right|^{6,20,21}$. Cryptochromes have been found in the retinas of migratory birds ${ }^{20,22-24}$ in various cell types that exhibit neuronal activity when the birds perform magnetic orientation ${ }^{22}$. Behavioural studies of fruit flies suggest that cryptochrome is required for magnetic sensing ${ }^{25-}$ ${ }^{31}$ but do not establish that it is the magnetically sensitive entity. Magnetic field effects on blue-light-dependent, cryptochrome-mediated growth responses in plants have been reported ${ }^{32}$ but were not replicated in an independent study $y^{33}$. Of the known photoreceptors, it appears that only cryptochromes and the closely related photolyases form radical pairs; since it was proposed 15 years ago 4 , cryptochrome remains the only candidate magnetoreceptor molecule.

Pronounced directional responses of a radical pair reaction to an Earth-strength magnetic field (ca. $50 \mu \mathrm{T}$ ) at physiological temperatures seem unlikely. Isotropic changes in reaction yields at the $1 \%$ level have been reported for cryptochrome and photolyase proteins in $1 \mathrm{mT}$ fields ${ }^{11,34}$ and theoretical predictions for (FAD ${ }^{\circ-}$ $\mathrm{Trp}^{\circ+}$ ) suggest that anisotropic effects at $50 \mu \mathrm{T}$ might be no larger than $0.1 \%{ }^{14}$. Until more is known about the signal transduction mechanism it is difficult to judge whether such small effects could form the basis of a viable compass ${ }^{6}$. However, it seems reasonable to assume that evolution would have availed itself 
of any opportunity to boost the sensitivity of the fundamental response in order to optimise the compass. One theoretical possibility (for which there is currently no biological evidence) is that the magnetic field experienced by a radical pair sensor could be amplified by nearby magnetic nanoparticles composed of biogenic magnetite ${ }^{35-37}$.

We present here experimental evidence for a chemical amplification mechanism in which slow reaction steps in the cryptochrome photocycle can significantly boost the response to a weak magnetic field. These enhancements, which have not been previously observed, were found for continuous illumination under conditions that allow long-lived reaction intermediates to accumulate. The details of the amplification process have been characterised through studies of intermolecular photoreactions of flavins and the photo-induced intramolecular radical pair, $\left(\mathrm{FAD}^{\bullet-} \operatorname{Trp}^{\bullet+}\right)$, in cryptochrome. We end by arguing that if such amplification occurred in a cryptochrome-based sensor in vivo, the detection sensitivity could be boosted by more than an order of magnitude.

\section{Results and discussion}

Magnetic field effects on intermolecular flavin photocycles. Magnetic field effects (MFEs) on flavin ( $F$ ) photochemistry are generally well-understood (Fig. 1 $)^{38-43}$. Photoexcitation of the fully oxidised state $\left(\mathrm{F} \rightarrow{ }^{1} \mathrm{~F}^{*}\right)$, is followed either by fluorescence $\left({ }^{1} \mathrm{~F}^{*} \rightarrow \mathrm{F}\right)$ or intersystem crossing $\left({ }^{1} \mathrm{~F}^{*} \rightarrow{ }^{3} \mathrm{~F}^{*}\right)$. Intermolecular electron transfer from a closed shell electron donor, $D$, to the triplet flavin gives a triplet radical pair, ${ }^{3}\left(\mathrm{~F}^{\bullet-} \mathrm{D}^{\bullet+}\right)$ which interconverts coherently with the corresponding singlet state, ${ }^{1}\left(\mathrm{~F}^{\bullet-} \mathrm{D}^{\circ+}\right)$ (curly arrows in Fig. 1). At the same time, (i) reverse electron transfer returns ${ }^{1}\left(F^{\bullet-} D^{\bullet+}\right)$ to the ground state reactants while (ii) $\mathrm{F}^{\bullet-}$ and $\mathrm{D}^{\bullet+}$ diffuse apart to form independent free radicals. Bulk re-encounter of $\mathrm{F}^{\bullet-}$ and $\mathrm{D}^{\bullet+}$ regenerates ${ }^{1}\left(\mathrm{~F}^{\bullet-} \mathrm{D}^{\bullet+}\right)$ and ${ }^{3}\left(\mathrm{~F}^{\bullet-} \mathrm{D}^{\bullet+}\right)$ in the statistical ratio of 1:3. In the absence of strong heavy-atom-mediated spin-orbit interactions all electron transfer steps conserve electron spin. The reaction steps labelled $k_{\mathrm{F}}$ and $k_{\mathrm{D}}$ in Fig. 1 are discussed below.

The sensitivity to applied magnetic fields lies in the conversion of ${ }^{3}\left(\mathrm{~F}^{\bullet-} \mathrm{D}^{\bullet+}\right)$ to ${ }^{1}\left(F^{\bullet-} D^{\bullet+}\right)$ which is driven by the magnetic (hyperfine) interactions of the electron spins with the spins of magnetic nuclei $\left({ }^{1} \mathrm{H}\right.$ and $\left.{ }^{14} \mathrm{~N}\right)$ and by the (Zeeman) interaction of the electron spins with applied magnetic fields. A magnetic field stronger than the hyperfine interactions $(\sim 3 \mathrm{mT})$ energetically isolates two of the three triplet sub-levels, inhibiting triplet $\rightarrow$ singlet conversion and therefore reducing the fraction of radical pairs that return directly to the ground state reactants. Reported MFEs ${ }^{38-40}$ and other spin-dependent phenomena ${ }^{44-46}$ are consistent with Fig. 1.

Under conditions of continuous, low-intensity photoexcitation, only the free radicals and the ground state reactants have appreciable concentrations. The other species have lifetimes less than a microsecond and are consequently present at much lower photostationary levels (see Supplementary Section C). When a strong magnetic field is switched on, and the return of radical pairs to the ground state becomes less efficient, the photocycle re-equilibrates to a state in which there are more free radicals and correspondingly fewer ground state 
molecules. Consequently, there is a reduction in the fluorescence of ${ }^{1} \mathrm{~F}^{*}$ whose concentration is proportional to that of the ground state $(\mathrm{F})$.

Flavin-lysozyme magnetic field effects. Initial experiments were conducted using intermolecular models of the intramolecular flavin-tryptophan radical pair in cryptochrome. Hen egg white lysozyme (a $14 \mathrm{kD}$ protein) was chosen as the electron donor because it is known to form radical pairs $\left(\mathrm{F}^{\circ-} \mathrm{D}^{\circ+}\right)$ in accord with Fig. 1, by electron transfer from a solvent-exposed Trp residue on the surface of the protein to photo-excited triplet states of flavins ${ }^{30,31}$. Fluorescence-detected MFEs for this photoreaction are shown in Fig. 2. An aqueous solution of flavin mononucleotide, FMN, and lysozyme was irradiated continuously with a $470 \mathrm{~nm}$ laser for a period of several minutes, monitoring the fluorescence all the while. Throughout the measurement a magnetic field was switched between $0 \mathrm{mT}$ and $27 \mathrm{mT}$ every $1.68 \mathrm{~s}$. Superimposed on a gradual (tens of seconds) decrease in fluorescence intensity (Fig. 2a) (which we attribute to a combination of reequilibration of the photocycle, photobleaching reactions and diffusion) is a small but distinct modulation in synchrony with the magnetic field steps. As the background fluorescence intensity drops, so does the amplitude of the fieldinduced modulations. That is, the relative change in the fluorescence does not change significantly with time. Fig. $2 b$ shows this variation averaged over a large number of field cycles. The data are presented as the fractional change in the intensity of the fluorescence relative to the field-off condition. As anticipated, switching the field on/off caused a reduction/increase in the fluorescence. Although the changes are relatively small, the signal-to-noise is excellent and the signals were highly reproducible.

Remarkably, the MFE in Fig. 2b shows biphasic kinetics. After switching the field on, a fast component ( $<1 \mathrm{~ms}$ ) was followed by a much slower phase with an exponential time constant, $\tau=470 \pm 10 \mathrm{~ms}$. The same pattern of fast and slow phases was seen when the field was switched off. The amplitudes of the two components were used to define prompt $\left(\chi_{\mathrm{p}}\right)$ and delayed $\left(\chi_{\mathrm{d}}\right)$ MFEs (Fig. $\left.2 \mathrm{~b}\right)$. The amplification factor, $E$, defined as $\chi_{\mathrm{d}} / \chi_{\mathrm{p}}$, was $2.320 \pm 0.007$. The fast component immediately following the field-jump is attributed to the attainment of a new steady state at a rate limited by the bulk re-encounter of the free radicals, $\mathrm{F}^{{ }^{-}-}$and $\mathrm{D}^{\circ+}$. The timescale of this process is estimated at $0.2-1.0 \mathrm{~ms}$, based on a diffusion controlled rate constant $\left(\sim 10^{9} \mathrm{M}^{-1} \mathrm{~s}^{-1}\right)$ and $1.0-5.0 \mu \mathrm{M}$ concentrations of $\mathrm{F}^{\circ-}$ and $\mathrm{D}^{\circ+}$. The slow components in Fig. $2 \mathrm{~b}$ were unexpected.

Flavin-tryptophan magnetic field effects. To see whether the magnetic field effects observed for FMN reacting with lysozyme were influenced by the electron donor being part of a large (protein) molecule, we studied the same photoreaction but with the tryptophan present as the free amino acid. Fig. 1 should still be appropriate but with different rate constants for some of the reaction steps. Similar, biphasic kinetics were observed (Figs. $2 \mathrm{~d}$ and e) except that the slow component of the fluorescence had the opposite phase, i.e. $\chi_{\mathrm{d}}<\chi_{\mathrm{p}}$ ( $E=0.644 \pm 0.003)$, and was an order of magnitude faster $(\tau=55 \pm 1 \mathrm{~ms})$ than for the Trp residues in lysozyme. For both FMN/lysozyme and FMN/tryptophan reactions there was no statistically significant difference between individual cycles (Figs $2 a$ and d) and their means (Figs $2 b$ and e, respectively). In both cases, the averaged responses to switching the field on and off had identical amplitudes and kinetics within experimental error. 
Dependence on magnetic field intensity. A clue to the origin of the slow phase comes from the dependence of its amplitude on the strength of the applied magnetic field. Fig. 2c shows the fluorescence-detected MFE for the FMN/lysozyme reaction measured at different times $(T=10-5000 \mathrm{~ms})$ after the field was switched on (see Methods and Supplementary Sections A and B for experimental details). As expected from Fig. $2 b$, the MFE became more negative with increasing $T$. The field, $B_{1 / 2}$, at which it reached $50 \%$ of its limiting value (obtained by Lorentzian fitting and extrapolation) was $6.4 \pm 0.2 \mathrm{mT}$. This number, which was found to be independent of $T$, is consistent with the hyperfine interactions in the flavin-Trp radical pair augmented by a contribution from electron spin relaxation ${ }^{11,40}$. The invariance of $B_{1 / 2}$ indicates that no other mechanisms, e.g. triplet-triplet or triplet-doublet pairs ${ }^{47,48}$, are involved. The wavelength-dependence of the fluorescence (see Supplementary Section B) is consistent with ${ }^{1} \mathrm{FMN} *$ being the sole source of both fast and slow components.

Slow radical termination reactions. The reaction scheme, as discussed above, cannot account for the slow phase in Figs $2 \mathrm{~b}$ and e. Simulations using independently determined values of the rate constants of the various steps in the photocycle (see Supplementary Section C), show that the fluorescence-detected MFE should be monophasic with a short time constant (ca. $100 \mu \mathrm{s}$ ) determined by the bulk re-encounter of $\mathrm{F}^{\circ-}$ and $\mathrm{D}^{\circ+}$. However, biphasic kinetics can be obtained by introducing additional slow reaction steps which return the free radicals to their respective ground states (coloured arrows in Fig. 1). These reactions, with rate constants $k_{\mathrm{F}}$ and $k_{\mathrm{D}}$, are assumed for simplicity to have first order kinetics. When $k_{\mathrm{F}}=k_{\mathrm{D}}$, the extra pathways do not affect the simulated kinetics which remain fast $\left(\sim 100 \mu \mathrm{s}\right.$, Fig. 3a left). However, when $k_{\mathrm{F}} \neq k_{\mathrm{D}}$, the prompt effect is followed by a slow phase. If $k_{\mathrm{D}} / k_{\mathrm{F}}>1$, the MFE is enhanced ( $E>1$, Fig. 3a, right); if $k_{\mathrm{D}} / k_{\mathrm{F}}<1$, the MFE is attenuated ( $E<1$, Fig. 3a, centre). In both cases, the timescale of the slow component is determined by the values of $k_{\mathrm{F}}$ and $k_{\mathrm{D}}$.

For a broad range of values of the two rate constants, $E$ was found to depend principally on the ratio $k_{\mathrm{D}} / k_{\mathrm{F}}$ (Fig. $3 \mathrm{~b}$ ). Surprisingly large steady-state enhancements can result from these very slow processes. For $k_{\mathrm{D}}$ and $k_{\mathrm{F}}$ in the range $10^{-1}$ to $10^{3} \mathrm{~s}^{-1}$, amplification factors of 5-20 can be realised using independently determined or estimated values for the other rate constants in the reaction scheme (Supplementary Section C). Lower light intensities were found to lead to larger enhancements (Supplementary Section C).

Rate constants. Incorporation of the additional radical termination reactions into the photocycle also allowed a quantitative analysis of the kinetics in Figs $2 \mathrm{~b}$ and $\mathrm{e}$ (Supplementary C). For the FMN/lysozyme case, we find $k_{\mathrm{F}}=0.70 \mathrm{~s}^{-1}$ and $k_{\mathrm{D}}=4.9$ $\mathrm{s}^{-1}$; and for FMN/Trp, $k_{\mathrm{F}}=70 \mathrm{~s}^{-1}$ and $k_{\mathrm{D}}=12 \mathrm{~s}^{-1}$. An approximate treatment of the kinetics (using a highly simplified version of the intermolecular reaction scheme, see Supplementary Section D) suggests that $E \approx \sqrt{k_{\mathrm{D}} / k_{\mathrm{F}}}$. For these two reactions $\sqrt{k_{\mathrm{D}} / k_{\mathrm{F}}}=2.6$ and 0.41 , respectively, which agree reasonably well with the experimental amplification factors: $E=2.320$ and 0.644 , respectively, as reported above.

The smaller value of $k_{\mathrm{D}}$ for lysozyme is presumably due to the lower accessibility of the Trp residues in the protein compared to the free amino acid ${ }^{49}$. The values of $k_{\mathrm{F}}$ for the two reactions can be understood as follows. The $\mathrm{FMNH}^{\bullet}$ radical has a 
$\mathrm{p} K_{\mathrm{a}}$ of $8.6^{50,51}$ so that there is a higher fraction of $\mathrm{FMN}^{--}$present in the $\operatorname{Trp}$ experiment $(\mathrm{pH} 6.2)$ than in the lysozyme case $(\mathrm{pH} \mathrm{4.0)}$. A likely contributor to the flavin radical termination reaction is oxidation by molecular oxygen which is $10^{3}$ $10^{4}$ times faster for $\mathrm{FMN}^{\bullet-}$ than for $\mathrm{FMNH}^{\bullet 50,51}$. Removal of flavin radicals should therefore be slower at lower $\mathrm{pH}$. In accord with this, reducing the $\mathrm{pH}$ of the FMN/Trp reaction from 6.2 to 2.7 changes the amplification factor from $E<1$ (Fig. 2e) to $E>1$ (Fig. 2f), consistent with a substantial reduction in $k_{\mathrm{F}}$.

Further observations. Further support for the importance of slow radical termination reactions comes from three additional observations made by changing experimental conditions in ways that were expected to alter the ratio of $k_{\mathrm{D}}$ to $k_{\mathrm{F}}$. First, addition of $50 \mu \mathrm{M}$ of the reductant tris(2-carboxyethyl)phosphine (TCEP) to the FMN/Trp reaction at pH 6.2 increased $E$ from 0.644 (Fig. 2e) to 0.89 \pm 0.02 (Fig. 2f) by increasing the rate of reduction of the Trp radical $\left(k_{\mathrm{D}}\right)$. Second, the presence of $20 \%$ glycerol boosted $E$ for the $\mathrm{FMN} /$ lysozyme reaction from 2.32 (Fig. 2b) to $3.55 \pm 0.02$ and changed $\tau$ from $470 \mathrm{~ms}$ to $2.2 \mathrm{~s}$ (Supplementary Section $\mathrm{B}$ ). The increase in solvent viscosity presumably reduces the diffusioncontrolled contributions to $k_{\mathrm{D}}$ and $k_{\mathrm{F}}$ in such a way that $k_{\mathrm{D}} / k_{\mathrm{F}}$ becomes larger. Third, increasing either the concentration of FMN or the light intensity resulted in a reduction in $E$ for the $\mathrm{FMN} / \mathrm{Trp}$ reaction implying a decrease in $k_{\mathrm{D}} / k_{\mathrm{F}}$. Both observations may be understood by considering the likely mechanisms of the $\mathrm{FH}^{\circ} \rightarrow \mathrm{F}$ oxidation step. One route for the removal of flavin radicals is disproportionation $\left(2 \mathrm{FH}^{\bullet} \rightarrow \mathrm{F}+\mathrm{FH}_{2}{ }^{50,51}\right)$. Another involves molecular oxygen $\left(\mathrm{FH}^{\bullet}\right.$ $+\mathrm{O}_{2} \rightarrow \mathrm{F}+\mathrm{O}_{2}^{--}+\mathrm{H}^{+}$, followed by the much faster reaction, $\mathrm{FH}^{\bullet}+\mathrm{O}_{2}^{--}+\mathrm{H}^{+} \rightarrow \mathrm{F}+$ $\mathrm{H}_{2} \mathrm{O}_{2}{ }^{50,51}$ ). Both processes result in a supra-linear dependence of the oxidation rate constant $\left(k_{\mathrm{F}}\right)$ on the flavin radical concentration because of the second order kinetics of the first and the autocatalytic nature of the second. The involvement of oxygen was confirmed by the observation of an increase in the amplification factor for de-oxygenated solutions, consistent with an increase in $k_{\mathrm{D}} / k_{\mathrm{F}}$ (see Supplementary Section E).

Origin of the amplification. The origin of the enhanced MFE when $k_{\mathrm{D}}>k_{\mathrm{F}}$ (right hand side of Fig. 3a, and Fig. 2b) may be understood as follows. When the magnetic field is switched on, the fluorescence initially decreases because of the increase in the concentration of free radicals, as explained above. On the completion of this rapid (submillisecond) phase, the concentrations of the free radicals $\left(\mathrm{F}^{\circ-}\right.$ and $\mathrm{D}^{\circ+}$ in Fig. 1 ) are equal. Then, as the $k_{\mathrm{F}}$ and $k_{\mathrm{D}}$ steps start to take effect ( $>10 \mathrm{~ms}$ ), the faster removal of $\mathrm{D}^{\circ+}$ (when $k_{\mathrm{D}}>k_{\mathrm{F}}$ ) causes its concentration to drop more rapidly than that of $\mathrm{F}^{\circ-}$. Consequently, the lifetime and therefore the steady state concentration of $\mathrm{F}^{{ }^{-}-}$both increase, the concentration of ground state flavin falls and so therefore does the fluorescence intensity. The opposite change occurs when $k_{\mathrm{D}}<k_{\mathrm{F}}$. The slow termination steps have a negligible effect on the magnetic sensitivity when measured under flash photolysis conditions because they cannot compete with the much faster radical-radical recombination reaction. This explanation of the amplification effect was corroborated by experiments using square-wave modulated light intensities, in the absence of a magnetic field, as an alternative way of shifting the position of the photo-stationary state (Supplementary Section F).

Flavin-ascorbic acid magnetic field effects. Next, we explored the amplification for the photoreaction of FMN with ascorbic acid. This electron donor was chosen because the radical pair it forms with FMN is expected to be considerably more 
sensitive to weak magnetic fields than $\left(\mathrm{FMN}^{\bullet-} \operatorname{Trp}^{\bullet+}\right)$ by virtue of the much smaller hyperfine interactions in the ascorbyl radical ${ }^{14}$. The magnetic field dependence of the fluorescence (Fig. 4a), recorded in the same manner as Fig. 2c, showed the biphasic behaviour that is typical of long-lived radical pairs. The positive MFE, with a peak intensity at approximately $0.7 \mathrm{mT}$ and a zero-crossing close to $1.4 \mathrm{mT}$, is known as the 'low field effect' ${ }^{52,53}$.

Figs $4 \mathrm{~b}$ and $\mathrm{c}$ show averaged fluorescence-detected MFEs for the FMN/ascorbic acid reaction with the field cycled between 0 and $27 \mathrm{mT}$ and between 0 and 0.7 $\mathrm{mT}$, respectively. As expected from Fig. 4a, the latter is weaker than the former and of opposite phase. Less predictably, the amplification factor is bigger for the weaker field: $E=1.98 \pm 0.02$ at $27 \mathrm{mT}$ and $5.6 \pm 0.3$ at $0.7 \mathrm{mT}$. The kinetics of the slow phase are also a little different: $\tau=170 \mathrm{~ms}$ at $27 \mathrm{mT}$ and $190 \mathrm{~ms}$ at $0.7 \mathrm{mT}$. The origin of both differences may again be understood in terms of the supralinear nature of the slow processes that oxidise $\mathrm{FH}^{\bullet}$. The prompt MFE at $27 \mathrm{mT}$ $(0.7 \mathrm{mT})$ leads to an increase (decrease) in the $\mathrm{FH}^{\circ}$ concentration and a disproportionate increase (decrease) in $k_{\mathrm{F}}$. This translates into a larger $E$ and a longer $\tau$ at $0.7 \mathrm{mT}$.

Cryptochrome photocycle. We now turn from bimolecular electron transfer reactions to the intramolecular photochemistry of cryptochrome (Fig. 5). Photoexcitation of the fully oxidised state of the FAD cofactor leads to a (FAD ${ }^{\circ-}$ $\mathrm{Trp}^{\bullet+}$ ) radical pair (termed RP1 in Ref. ${ }^{11}$ ) in which Trp ${ }^{\bullet+}$ is the oxidised form of the terminal residue of the Trp-triad electron transfer chain. One difference from Fig. 1 is that intra-protein electron transfer is markedly faster than intersystem crossing ( $\left({ }^{1} \mathrm{FAD}^{*} \rightarrow{ }^{3} \mathrm{FAD}^{*}\right)$ and produces the radical pair in an initial singlet state ${ }^{11,}$ ${ }^{34,54}$. The singlet state of the radical pair either undergoes reverse electron transfer to regenerate the ground states of the flavin and the Trp or is converted coherently into the triplet radical pair state. But there the correspondence to Fig. 1 ends. Instead of diffusive separation of the radicals (impossible in the protein), one or both of $\mathrm{FAD}^{\circ-}$ and $\mathrm{Trp}^{\circ+}$ changes its protonation state to give a longer lived, magnetically insensitive radical pair (denoted RP2 in Ref. ${ }^{11}$ ). The constituents of $\mathrm{RP} 2$ are then independently returned to their respective ground states by slow redox reactions analogous to the $k_{\mathrm{F}}$ and $k_{\mathrm{D}}$ processes in Fig. 1 . Despite the differences in the two reaction schemes, amplification of the magnetic field effect $(E>0)$ is expected for the intermolecular photocycle provided $k_{\mathrm{D}}>k_{\mathrm{F}}$ (Supplementary Sections C and D).

Cryptochrome magnetic field effects. Fig. 6a shows the difference between the fluorescence spectrum of Arabidopsis thaliana cryptochrome (AtCry1) with and without a $12.2 \mathrm{mT}$ magnetic field. The signal grew dramatically in strength as the delay, $T$, between applying the field and recording the fluorescence was increased from $0.5 \mathrm{~s}$ to $3.5 \mathrm{~s}$. All such spectra closely matched the fluorescence spectrum recorded without an applied field demonstrating that the only magnetically sensitive luminescent state is ${ }^{1} F A D^{*}$. Changes in the time-dependence of the fluorescence produced by magnetic field cycling are shown in Fig. $6 \mathrm{~b}$. These signals are much weaker than for the intermolecular reactions (above) for two main reasons. First, the picosecond electron transfer in cryptochrome results in a low fluorescence quantum yield ${ }^{55,56}$. Second, the $k_{\mathrm{F}}$ and $k_{\mathrm{D}}$ steps are much slower than for the FMN reactions (Figs 2 and 4) meaning that even with the low light intensities used for these measurements there is a substantial depletion of the ground state and hence a reduction in the fluorescence intensity. Fig. $6 \mathrm{~b}$ shows an 
enhanced MFE growing in over several seconds ( $\tau=3.4 \pm 0.9 \mathrm{~s}$ ). Addition of 50 $\mu \mathrm{M}$ TCEP to accelerate the reduction of the Trp ${ }^{\bullet+}$ radical (Fig. $6 \mathrm{c}$ ) boosted the signal and caused it to rise more rapidly $(\tau=1.7 \pm 0.1 \mathrm{~s})$. In addition, a small prompt MFE was present (in Fig. 6c more clearly than in Fig. 6b).

We attribute the slow components in Figs $6 \mathrm{~b}$ and $\mathrm{c}$ to the radical termination steps in cryptochrome with $k_{\mathrm{D}}>k_{\mathrm{F}}$, as for FMN/lysozyme (Fig. 2b) but with opposite phase to FMN/lysozyme because the initial state of the radical pair in the protein is singlet rather than triplet. The small prompt MFE is assigned to the photoreaction of a small amount of free FAD with Trp residues on the surface of the protein ${ }^{44}$. This signal, which (as expected) has the same phase as the prompt MFE seen for FMN/lysozyme obscures any prompt MFE from the cryptochrome. There is no evidence that free FAD leads to an intermolecular delayed MFE: any such effect would occur with faster kinetics than seen in Fig. 6b. A kinetic analysis of the cryptochrome photocycle in Fig. 5 (see Supplementary Sections $C$ and D) shows that substantial enhanced MFEs can be anticipated when $k_{\mathrm{D}}>k_{\mathrm{F}}$.

Cryptochrome magnetic signalling. The data in Fig. 6a-c demonstrate a substantial amplification of the magnetic field effect on the cryptochrome photocycle, using FAD fluorescence as a direct indicator of the concentration of the ground state of the protein. To relate these findings to the putative role of cryptochrome in the avian magnetic compass, it is important to establish whether there is also an amplification effect on the signalling state of the protein. Although almost nothing is known about magnetic signal transduction by cryptochromes, it seems unlikely that the ground state of the protein is responsible. We presume that the magnetically sensitive radical pair state $\left(\sim 1 \mu\right.$ s lifetime $\left.{ }^{11}\right)$ leads to a longer lived species - for example, the stabilised radical pair state RP2 characterized by Maeda et al. ( $100 \mu$ s lifetime $)^{11}$ - which could, in turn, give rise to a signalling state. It seems plausible that the latter would be further stabilised against reverse electron transfer by reduction of the Trp radical by an exogenous electron donor. In terms of the reactions studied here, this situation corresponds to $k_{\mathrm{D}}>k_{\mathrm{F}}$ and therefore an amplification factor $E>1$. The question is therefore whether the magnetic field effect on the radical form of the protein is amplified by the asymmetry in the slow radical termination reactions.

Although we have presented relative (percentage) magnetic field effects in Figs 2 , 4 and 6 , this is probably not the relevant quantity for magnetic signalling: even a large relative change in the steady state concentration of the signalling state is unlikely to form the basis of a magnetic compass if that concentration is tiny. The quantity that determines the signal-to-noise ratio of the sensor is more likely to be the absolute change in the concentration of the radicals present under steady state conditions. This is the basis of the analysis of magnetoreceptor sensitivity by Weaver et al. $^{57}$ who considered the change in the absolute number of neurotransmitter molecules supposedly formed from the radical pair state. We therefore calculated the prompt and steady state changes in the concentration of the FAD-radical form the protein as a function of $k_{\mathrm{D}}$ and $k_{\mathrm{F}}$ using the reaction scheme in Fig. 5 and a kinetic analysis described in the Supplementary Section C. The results are shown in Fig. 7. The largest delayed (steady state) field-induced concentration changes occur when $k_{\mathrm{D}} \gg k_{\mathrm{F}}$ and are substantially larger than the prompt effect for any values of $k_{\mathrm{D}}$ and $k_{\mathrm{F}}$. Furthermore, when the light intensity is reduced, a larger ratio $k_{\mathrm{D}} / k_{\mathrm{F}}$ is necessary to achieve a significant amplification 
(see Supplementary Section C). That is, the lower the light intensity the more important is the amplification phenomenon.

\section{Conclusions}

Slow radical termination reactions have been shown to lead to a significant amplification of the effects of weak magnetic fields on flavin-containing radical pairs under conditions of continuous photoexcitation. That these enhancements have not previously come to light is probably because most studies have either been conducted with pulsed illumination ${ }^{11,58}$ or have used magnetic fields that were amplitude-modulated at frequencies faster than the slow reaction steps ${ }^{59}$. The magnitude of the amplification increases with the ratio $\left(k_{\mathrm{D}} / k_{\mathrm{F}}\right)$ of the rate constants for the oxidation of the flavin radical $\left(k_{\mathrm{F}}\right)$ and the reduction of the counter-radical $\left(k_{\mathrm{D}}\right)$. Significant amplification factors $(E)$ have been observed for cryptochrome and the related intermolecular photochemistry of free flavin molecules. The amplification factors are larger in weaker fields.

We see no obvious reason why the chemical amplification mechanism studied here could not function in a cryptochrome magnetoreceptor. In vivo photoexcitation of cryptochromes leads to accumulation of states of the protein that contain reduced forms of $F A D^{60}$ implying efficient reduction of the donor radical and hence substantial enhancement of the magnetic field effect (i.e. $k_{D} \gg$ $k_{\mathrm{F}}$ and hence $E \gg>1$ ). It has been suggested that the signalling state of the putative cryptochrome magnetosensor contains FAD that is either singly reduced (FAD ${ }^{\circ-}$ or FADH $\left.{ }^{\circ}\right)^{61}$ or, after further photoexcitation, fully reduced $\left(\mathrm{FADH}^{-}\right)^{12,13}$. Either way, rapid reduction of the donor-radical would stabilise the signalling state against reverse electron transfer so promoting signal transduction. There would appear to be ample scope for evolution to have optimised the amplification by tuning $k_{\mathrm{D}}$ and $k_{\mathrm{F}}$ via factors such as the redox potentials of the flavin and donor radicals and their accessibility to, and the local concentrations of, intracellular oxidants and reductants. We suggest that substantial chemical amplification of radical pair magnetic field effects could have a significant impact on the viability of a cryptochrome-based magnetic compass sensor.

\section{Methods}

Spectrally-resolved fluorescence measurements. Square-wave-modulated magnetic fields of up to $12 \mathrm{mT}$ (rise time $3 \mathrm{~ms}$ ) were generated using Helmholtz coils (diameter $3.6 \mathrm{~cm}$ ). Samples positioned between the coils were irradiated with one of two continuous-wave diode lasers ( $405 \mathrm{~nm}$ and $450 \mathrm{~nm}$ ); where appropriate the excitation power was attenuated by neutral density filters. Fluorescence was dispersed by a spectrograph onto a charge-coupled device (CCD) camera where the fluorescence was integrated for a period of $20-500 \mathrm{~ms}$ following a set delay, $T$, after switching on the field. 35-900 measurements were averaged for each magnetic field strength in a randomized order. Experiments were conducted at the following laser powers, laser wavelengths and sample temperatures: Fig. 2c: $270 \mathrm{~mW}, 405 \mathrm{~nm}, 22^{\circ} \mathrm{C}$; Fig. $4 \mathrm{a}: 350 \mathrm{~mW}, 405 \mathrm{~nm}, 22^{\circ} \mathrm{C}$; Fig. 6a: $1 \mathrm{~mW}, 450 \mathrm{~nm}, 5^{\circ} \mathrm{C}$. 
Time-resolved fluorescence measurements. The data shown in Figs $2 a, 2 b, 2 d-f$, $4 b, 4 c, 6 b$, and $6 c$ were acquired using an inverted fluorescence microscope.

Samples (optical path length $0.5 \mathrm{~mm}$, depth of field $0.8 \mu \mathrm{m}$ ) were epi-illuminated with the attenuated and expanded beam of a $470 \mathrm{~nm}, 80 \mathrm{~mW}$ diode laser focussed at the back aperture of an oil immersion objective. Fluorescence was collected through the same objective, and recorded using an electron-multiplying CCD camera. Magnetic fields (up to $27 \mathrm{mT},<70 \mu$ s rise time) were generated by a solenoid wound around a ferrite core (diameter $3 \mathrm{~mm}$ ) fixed in a non-metallic mount along the optical axis, placed $1 \mathrm{~mm}$ above the sample. For Figs 2 and 4 , the camera exposure time was $5 \mathrm{~ms}$ per frame and the interval between frames was 7 ms. Typically 16,000 frames were recorded with the field stepped between zero and a preset value every 80,160 or 240 frames. For Fig. 6, 10,000 frames were recorded with a camera exposure time of $40 \mathrm{~ms}$ per frame, a frame interval of 42 $\mathrm{ms}$, with the field switched every 100 frames. For the cryptochrome measurements, the diode laser output was highly attenuated (to $0.1 \mathrm{~mW}$ ) and the stage cooled to $-5^{\circ} \mathrm{C}$ (sample temperature approx. $2{ }^{\circ} \mathrm{C}$ ) to minimize sample photodegradation. All other measurements were performed at $22^{\circ} \mathrm{C}$.

Sample preparation. AtCry1 was expressed in Nicotina benthamiana (Planet Biotechnology, Hayward, California). During sample preparation and the final purification step, protein solutions were shielded from ambient light and kept at temperatures below $4{ }^{\circ} \mathrm{C}$. For the fluorescence measurements, AtCry1 (ca. $100 \mu \mathrm{M}$ ) was contained in $10 \mathrm{mM}$ Tris, $0.15 \mathrm{mM} \mathrm{NaCl}, 30 \%$ glycerol v/v, pH 7.4). The concentration of the flavin was verified spectrophotometrically. All studies were conducted on freshly prepared samples.

Modelling amplification effects. Details of the simulation methods, both for interand intramolecular flavin-containing radical pairs, are described in the Supplementary Sections C and D.

\section{Acknowledgements}

We thank Neville Baker for expert technical assistance. E.W.E. is indebted to EPSRC and SABMiller plc for his doctoral scholarship. C.A.D. gratefully acknowledges her current Imperial College Junior Research Fellowship. We are grateful to the following for financial support: the Defense Advanced Research Projects Agency (QuBE: N66001-10-1-4061), the European Research Council (under the European Union's 7th Framework Programme, FP7/2007-2013/ ERC grant agreement no. 340451), the Air Force Office of Scientific Research (Air Force Materiel Command, USAF award no. FA9550-14-1-0095), and the EMF Biological Research Trust.

\section{Author contributions}

D.K. and E.W.E. contributed equally to this work. D.K., E.W.E. and V.D. designed and performed the experiments. D.K. and E.W.E. analysed the data. C.A.D. advised on the production of AtCry1 samples. M.I.W. helped oversee the fluorescence microscopy experiments. C.R.T., S.R.M. and P.J.H. coordinated the study. P.J.H., D.K. and E.W.E. wrote the paper. All authors discussed the results and commented on the manuscript. 


\section{Additional information}

Supplementary information is available in the online version of the paper.

\section{Competing financial interests}

The authors declare no competing financial interests.

\section{Author for correspondence}

Correspondence and requests for materials should be addressed to peter.hore@chem.ox.ac.uk. 


\section{Figure captions}

Figure 1 | Intermolecular radical pair reactions of flavins.

Reaction scheme for the intermolecular photochemical reaction of a flavin $(F)$ with an electron donor (D). The radicals, $\mathrm{F}^{\bullet-}$ and $\mathrm{D}^{\bullet+}$, are either charged as shown or (de)protonated depending on the $\mathrm{pH}$. The slow reaction steps labelled with the rate constants $k_{\mathrm{D}}$ and $k_{\mathrm{F}}$ are responsible for the amplification of the magnetic field effect. The other reaction steps are as follows: photoexcitation of the ground state flavin $(\mathrm{F})$ to its first excited singlet state $\left({ }^{1} \mathrm{~F}^{*}\right)$; fluorescence of ${ }^{1} \mathrm{~F}^{*}$; intersystem crossing from ${ }^{1} \mathrm{~F}^{*}$ to the excited triplet state of the flavin $\left({ }^{3} \mathrm{~F}^{*}\right)$; spinconserving electron transfer from the donor $\mathrm{D}$ to ${ }^{3} \mathrm{~F}^{*}$ to form the triplet state of the radical pair, ${ }^{3}\left(\mathrm{~F}^{\bullet-} \mathrm{D}^{\bullet+}\right)$; coherent interconversion of the triplet and singlet, ${ }^{1}\left(\mathrm{~F}^{\bullet-} \mathrm{D}^{\bullet+}\right)$, radical pair states driven by hyperfine and Zeeman magnetic interactions (curly arrows); spin-allowed reverse electron transfer within the singlet radical pair which regenerates the ground state of both reactants; diffusive separation to form free radicals which subsequently re-encounter to form radical pairs. Singlet-triplet interconversion becomes less efficient in a magnetic field stronger than the hyperfine interactions because only the $T_{0}$ triplet sub-state is able to mix with the singlet whereas $T_{+1}$ and $T_{-1}$ as well as $T_{0}$ mix with the singlet when the external magnetic field is weaker than the hyperfine interactions.

\section{Figure 2. Amplified magnetic field effects in intermolecular reactions of flavins.}

a, Fluorescence intensity of an aqueous solution ( $\mathrm{pH} 4.0)$ of FMN $(10 \mu \mathrm{M})$ and lysozyme $(0.5 \mathrm{mM})$ as a function of the time after the onset of continuous illumination $(470 \mathrm{~nm})$ while switching an applied magnetic field between 0 and 27 $\mathrm{mT}$ every $1.68 \mathrm{~s}$. Modulation of the fluorescence intensity, shown expanded in the inset, occurs in synchrony with the magnetic field switching.

b. Average of the responses in a to one cycle of the magnetic field showing a prompt MFE $\left(\chi_{\mathrm{p}}\right)$ and a slowly established delayed $\operatorname{MFE}\left(\chi_{\mathrm{d}}\right)$.

c, Dependence of the MFE on the magnetic field strength for different delay times between switching the field on and measuring the fluorescence. The dashed lines are Lorentzian fits to the data used to determine the $B_{1 / 2}$ values.

d, As a but for a solution (pH 6.2) of FMN $(10 \mu \mathrm{M})$ and $\operatorname{Trp}(1.0 \mathrm{mM})$. The magnetic field was switched between 0 and $27 \mathrm{mT}$ every $0.56 \mathrm{~s}$.

e, Average of the responses in $\mathbf{d}$ to one cycle of the magnetic field. In contrast to b, the delayed MFE $\left(\chi_{\mathrm{d}}\right)$ is smaller than the prompt effect $\left(\chi_{\mathrm{p}}\right)$.

f, As e but (blue) with the $\mathrm{pH}$ reduced to 2.7 (citric acid buffer) and (orange) with $50 \mu \mathrm{M}$ TCEP ( $\mathrm{pH}$ 6.2), showing that the amplification factor can be changed by altering the rate constants $k_{\mathrm{D}}$ and $k_{\mathrm{F}}$.

The data in the field-on and field-off periods in $\mathbf{a}, \mathbf{b}, \mathbf{d}$ and $\mathbf{e}$ are shown in green and blue, respectively. The timing of the magnetic field steps is shown in grey at 
the top of panels $\mathbf{b}, \mathbf{e}$ and $\mathbf{f}$ ( $B_{0}$ is the magnetic field strength). $\ln \mathbf{b}$ and $\mathbf{e}$, the slow background decay of the fluorescence (visible in $\mathbf{a}$ and $\mathbf{d}$ ) has been subtracted.

\section{Figure 3. | Characteristics of the amplified magnetic field effect.}

a, Response of the concentration of the flavin excited state, ${ }^{1} \mathrm{~F}^{*}$, to sudden on/off switching of an external magnetic field $\left(B_{0}\right)$. Left: $k_{\mathrm{F}}=k_{\mathrm{D}}(E=1)$; centre: $k_{\mathrm{D}} / k_{\mathrm{F}}<1(E<1)$; right: $k_{\mathrm{D}} / k_{\mathrm{F}}>1(E>1)$. Depending on the ratio of their rate constants $\left(k_{\mathrm{D}} / k_{\mathrm{F}}\right)$, the radical termination reactions lead to a slow kinetic phase that enhances or attenuates the prompt magnetic field effect.

b, Contour plot of the amplification factor, $E$, as a function of the slow radical termination rate constants $k_{\mathrm{F}}$ and $k_{\mathrm{D}}$. $E$ depends principally on the ratio $k_{\mathrm{D}} / k_{\mathrm{F}}$; the largest amplification factors occur when $k_{\mathrm{D}} \gg k_{\mathrm{F}}$. The simulations were performed for an initial triplet radical pair and a strong magnetic field. The values of the other rate constants used in the simulation are given in Supplementary Section C.

\section{Figure 4. | Amplified magnetic field effects in the low-field region.}

a, Dependence of the MFE on the magnetic field strength for an aqueous solution $(\mathrm{pH} 3.8)$ of FMN $(10 \mu \mathrm{M})$ and ascorbic acid $(0.5 \mathrm{mM})$ with a $50 \mathrm{~ms}$ delay time between switching the field on and measuring the fluorescence. The positive MFE at fields below $1.4 \mathrm{mT}$ is known as the Low Field Effect; it is particularly prominent in this case because of the small hyperfine interactions in the ascorbyl radical.

b, Average of the responses to one cycle of a magnetic field switched between 0 and $27 \mathrm{mT}$ every $1.12 \mathrm{~s}$.

c, As b but for a $0.7 \mathrm{mT}$ magnetic field (in the region of the Low Field Effect). The prompt and delayed components have opposite phases in $\mathbf{b}$ and $\mathbf{c}$ because of the biphasic form of the magnetic field effect (shown in a).

The black lines in $\mathbf{b}$ and $\mathbf{c}$ are fits to single exponentials.

\section{Figure 5. Intramolecular radical pair reactions of cryptochromes.}

Reaction scheme for the intramolecular photochemistry of the FAD (F) and the terminal residue of the Trp-triad in cryptochrome as the electron donor (D). RP2 is a FAD-Trp radical pair in which the flavin radical $\left(\mathrm{F}^{\circ-}\right)$ is protonated or the Trp radical $\left(D^{\circ+}\right)$ is deprotonated or both; it is not magnetically sensitive. The slow steps labelled with the rate constants $k_{\mathrm{D}}$ and $k_{\mathrm{F}}$ are responsible for the amplification of the magnetic field effect. As shown, we assume that the flavin radical may be oxidised before or after the Trp radical is reduced. 


\section{Figure 6. | Amplified magnetic field effects in a cryptochrome.}

a, Magnetic field-induced changes in the fluorescence spectrum of an aqueous solution of AtCry1 (ca. $100 \mu \mathrm{M}, 10 \mathrm{mM}$ Tris, pH 7.4, $150 \mathrm{mM} \mathrm{NaCl}, 30 \%$ glycerol). Spectra (smoothed using a $25 \mathrm{~nm}$ moving average filter) are shown for different delay times, $T$, after switching on a $12.2 \mathrm{mT}$ magnetic field, having subtracted the zero-field spectrum. The magnetic field effect becomes dramatically stronger as $T$ is increased from $0.5 \mathrm{~s}$ to $3.5 \mathrm{~s}$. The black trace is the fluorescence spectrum measured in the absence of a magnetic field and scaled to match the maximum of the difference spectrum at $T=3.5 \mathrm{~s}$ (red trace).

b, Average of the responses of an aqueous solution of AtCry1 (ca. $100 \mu \mathrm{M}, \mathrm{pH}$ 7.4) to one cycle of a magnetic field switched between 0 and $27 \mathrm{mT}$ every $4.2 \mathrm{~s}$. The enhanced magnetic field effect grows over a period of several seconds as a result of the slow radical termination reactions $\left(k_{\mathrm{D}}>k_{\mathrm{F}}\right)$.

c, As $\mathbf{b}$ with the addition of TCEP $(50 \mu \mathrm{M})$. Accelerated reduction of the Trp ${ }^{\bullet+}$ radical by the TCEP boosts the magnetic field effect $\left(k_{\mathrm{D}}>>k_{\mathrm{F}}\right)$ and causes it to grow more rapidly.

In $\mathbf{b}$ and $\mathbf{c}$, the black lines are fits of the data to single exponentials assuming symmetric responses to switching the field on and off.

\section{Figure 7. | Simulations of magnetic-field induced changes in radical concentrations.}

a, Density plot of the absolute prompt change in the total concentration of flavin radicals produced by an applied magnetic field, plotted as a function of the radical termination rate constants $k_{\mathrm{F}}$ and $k_{\mathrm{D}}$.

b, As a, but showing the absolute steady state magnetic field-induced change.

The scale bar refers to both plots. The amplification factor in the centre of the yellow region of $\mathbf{b}$, where the absolute steady state change is largest, is 33 . 


\section{References}

1. Wiltschko R. \& Wiltschko W. Avian magnetic compass: Its functional properties and physical basis. Curr. Zool. 56, 265-276 (2010).

2. Mouritsen H. The Magnetic Senses. In: Galizia C. G., Lledo P.-M. (eds). Neurosciences - from Molecule to Behavior: a University Textbook. Springer-Verlag Berlin, 2013, pp 427-443.

3. Kishkinev D. A. \& Chernetsov N. S. Magnetoreception systems in birds: a review of current research. Biol. Bull. Rev. 5, 46-62 (2015).

4. Ritz T., Adem S. \& Schulten K. A model for photoreceptor-based magnetoreception in birds. Biophys. J. 78, 707-718 (2000).

5. Rodgers C. T. \& Hore P. J. Chemical magnetoreception in birds: a radical pair mechanism. Proc. Natl. Acad. Sci. USA 106, 353-360 (2009).

6. Mouritsen H. \& Hore P. J. The magnetic retina: light-dependent and trigeminal magnetoreception in migratory birds. Curr. Opin. Neurobiol. 22, 343-352 (2012).

7. Chaves I., et al. The cryptochromes: blue light photoreceptors in plants and animals. Annu. Rev. Plant Biol. 62, 335-364 (2011).

8. Giovani B., Byrdin M., Ahmad M. \& Brettel K. Light-induced electron transfer in a cryptochrome blue-light photoreceptor. Nature Struct. Biol. 10, 489-490 (2003).

9. Zeugner A., et al. Light-induced electron transfer in Arabidopsis cryptochrome-1 correlates with in vivo function. J. Biol. Chem. 280, 1943719440 (2005).

10. Biskup T., et al. Direct observation of a photoinduced radical pair in a cryptochrome blue-light photoreceptor. Angew. Chem. Internat. Ed. 48, 404-407 (2009).

11. Maeda K., et al. Magnetically sensitive light-induced reactions in cryptochrome are consistent with its proposed role as a magnetoreceptor. Proc. Natl. Acad. Sci. USA 109, 4774-4779 (2012).

12. Niessner C., et al. Magnetoreception: activated cryptochrome 1a concurs with magnetic orientation in birds. J. Roy. Soc. Interface 10, 20130638 (2013).

13. Niessner C., Denzau S., Peichl L., Wiltschko W. \& Wiltschko R. Magnetoreception in birds: I. Immunohistochemical studies concerning the cryptochrome cycle. J. Exp. Biol. 217, 4221-4224 (2014).

14. Lee A. A., et al. Alternative radical pairs for cryptochrome-based magnetoreception. J. Roy. Soc. Interface, 20131063 (2014).

15. Hogben H. J., Efimova O., Wagner-Rundell N., Timmel C. R. \& Hore P. J. Possible involvement of superoxide and dioxygen with cryptochrome in avian magnetoreception: origin of Zeeman resonances observed by in vivo EPR spectroscopy. Chem. Phys. Lett. 480 118-122 (2009).

16. Solov'yov I. A. \& Schulten K. Magnetoreception through cryptochrome may involve superoxide. Biophys. J. 96, 4804-4813 (2009).

17. Conrad K. S., Manahan C. C. \& Crane B. R. Photochemistry of flavoprotein light sensors. Nat. Chem. Biol. 10, 801-809 (2014).

18. Gao J., et al. Trp triad-dependent rapid photoreduction is not required for the function of Arabidopsis CRY1. Proc. Natl. Acad. Sci. U.S.A. 112, 91359140 (2015).

19. Steiner U. E. \& Ulrich T. Magnetic field effects in chemical kinetics and related phenomena. Chem. Rev. 89, 51-147 (1989). 
20. Liedvogel M. \& Mouritsen H. Cryptochromes-a potential magnetoreceptor: what do we know and what do we want to know? J. Roy. Soc. Interface 7, S147-S162 (2010).

21. Dodson C. A., Hore P. J. \& Wallace M. I. A radical sense of direction: signalling and mechanism in cryptochrome magnetoreception. Trends Biochem. Sci. 38, 435-446 (2013).

22. Mouritsen H., et al. Cryptochromes and neuronal-activity markers colocalize in the retina of migratory birds during magnetic orientation. Proc. Natl. Acad. Sci. USA 101, 14294-14299 (2004).

23. Möller A., Sagasser S., Wiltschko W. \& Schierwater B. Retinal cryptochrome in a migratory passerine bird: a possible transducer for the avian magnetic compass. Naturwissenschaften 91, 585-588 (2004).

24. Niessner C., et al. Avian ultraviolet/violet cones identified as probable magnetoreceptors. Plos One 6, e20091 (2011).

25. Gegear R. J., Casselman A., Waddell S. \& Reppert S. M. Cryptochrome mediates light-dependent magnetosensitivity in Drosophila. Nature 454, 1014-1018 (2008).

26. Yoshii T., Ahmad M. \& Helfrich-Forster C. Cryptochrome mediates lightdependent magnetosensitivity of Drosophila's circadian clock. PLoS Biol. 7, 813-819 (2009).

27. Gegear R. J., Foley L. E., Casselman A. \& Reppert S. M. Animal cryptochromes mediate magnetoreception by an unconventional photochemical mechanism. Nature 463, 804-807 (2010).

28. Foley L. E., Gegear R. J. \& Reppert S. M. Human cryptochrome exhibits light-dependent magnetosensitivity. Nature Comm. 2, (2011).

29. Fedele G., et al. Genetic analysis of circadian responses to low frequency electromagnetic fields in Drosophila melanogaster. PLoS Genet. 10, e1004804 (2014).

30. Fedele G., Green E. W., Rosato E. \& Kyriacou C. P. An electromagnetic field disrupts negative geotaxis in Drosophila via a CRY-dependent pathway. Nat Commun 5, (2014).

31. Marley R., Giachello C. N. G., Scrutton N. S., Baines R. A. \& Jones A. R. Cryptochrome-dependent magnetic field effect on seizure response in Drosophila larvae. Sci. Rep. 4, 5799 (2014).

32. Ahmad M., Galland P., Ritz T., Wiltschko R. \& Wiltschko W. Magnetic intensity affects cryptochrome-dependent responses in Arabidopsis thaliana. Planta 225, 615-624 (2007).

33. Harris S.-R., et al. Effect of magnetic fields on cryptochrome-dependent responses in Arabidopsis thaliana J. Roy. Soc. Interface 6, 1193-1205 (2009).

34. Henbest K. B., et al. Magnetic-field effect on the photoactivation reaction of Escherichia coli DNA photolyase. Proc. Natl. Acad. Sci. USA 105, 1439514399 (2008).

35. Binhi V. N. Stochastic dynamics of magnetosomes and a mechanism of biological orientation in the geomagnetic field. Bioelectromagnetics 27, 58-63 (2006).

36. Winklhofer M. \& Kirschvink J. L. A quantitative assessment of torquetransducer models for magnetoreception. J. Roy. Soc. Interface 7, S273S289 (2010).

37. Cai J. Quantum probe and design for a chemical compass with magnetic nanostructures. Phys. Rev. Lett. 106, 100501 (2011). 
38. Miura T., Maeda K. \& Arai T. Effect of coulomb interaction on the dynamics of the radical pair in the system of flavin mononucleotide and hen egg-white lysozyme (HEWL) studied by a magnetic field effect. J. Phys. Chem. B 107, 6474-6478 (2003).

39. Murakami M., Maeda K. \& Arai T. Dynamics of intramolecular electron transfer reaction of FAD studied by magnetic field effects on transient absorption spectra. J. Phys. Chem. A 109, 5793-5800 (2005).

40. Neil S. R. T., et al. Broadband cavity-enhanced detection of magnetic field effects in chemical models of a cryptochrome magnetoreceptor. J. Phys. Chem. B 118, 4177-4184 (2014).

41. Evans E. W., et al. Sensitive fluorescence-based detection of magnetic field effects in photoreactions of flavins. Phys. Chem. Chem. Phys. 17, 18456-18463 (2015).

42. Dodson C. A., et al. Fluorescence-detected magnetic field effects on radical pair reactions from femtolitre volumes. Chem. Commun. 51, 80238026 (2015).

43. Beardmore J. P., Antill L. M. \& Woodward J. R. Optical absorption and magnetic field effect based imaging of transient radicals. Angew. Chem. 54, 8494-8497 (2015).

44. Hore P. J. \& Broadhurst R. W. Photo-CIDNP of biopolymers. Prog. Nucl. Magn. Reson. Spectrosc. 25, 345-402 (1993).

45. Morozova O. B., et al. Time resolved CIDNP study of electron transfer reactions in proteins and model compounds. Mol. Phys. 100, 1187-1195 (2002).

46. Mok K. H. \& Hore P. J. Photo-CIDNP NMR methods for studying protein folding. Methods 34, 75-87 (2004).

47. Atkins P. W. \& Evans G. T. Magnetic-field effects on chemiluminescent fluid solutions. Mol. Phys. 29, 921-935 (1975).

48. Mani T. \& Vinogradov S. A. Magnetic field effects on triplet-triplet annihilation in solutions: modulation of visible/NIR luminescence. J. Phys. Chem. Lett. 4, 2799-2804 (2013).

49. Hore P. J. \& Kaptein R. Proton nuclear magnetic-resonance assignments and surface accessibility of tryptophan residues in lysozyme using photochemically induced dynamic nuclear polarization spectroscopy. Biochemistry 22, 1906-1911 (1983).

50. Vaish S. P. \& Tollin G. Flash photolysis of flavins. V. Oxidation and disproportionation of flavin radicals. J. Bioenerg. 2, 61-72 (1971).

51. Massey V. Activation of molecular-oxygen by flavins and flavoproteins. J. Biol. Chem. 269, 22459-22462 (1994).

52. Brocklehurst B. Spin correlation in geminate recombination of radical ions in hydrocarbons. 1. Theory of magnetic-field effect. J. Chem. Soc. Faraday Trans. II 72, 1869-1884 (1976).

53. Timmel C. R., Till U., Brocklehurst B., McLauchlan K. A. \& Hore P. J. Effects of weak magnetic fields on free radical recombination reactions. Mol. Phys. 95, 71-89 (1998).

54. Weber S., et al. Origin of light-induced spin-correlated radical pairs in cryptochrome. J. Phys. Chem. B 114, 14745-14754 (2010).

55. Song S. H., et al. Absorption and fluorescence spectroscopic characterization of cryptochrome 3 from Arabidopsis thaliana. J. Photochem. Photobiol. B 85, 1-16 (2006). 
56. Shirdel J., Zirak P., Penzkofer A., Breitkreuz H. \& Wolf E. Absorption and fluorescence spectroscopic characterisation of the circadian blue-light photoreceptor cryptochrome from Drosophila melanogaster (dCry). Chem. Phys. 352, 35-47 (2008).

57. Weaver J. C., Vaughan T. E. \& Astumian R. D. Biological sensing of small field differences by magnetically sensitive chemical reactions. Nature 405, 707-709 (2000).

58. Maeda K., et al. Following radical pair reactions in solution: a step change in sensitivity using cavity ring-down detection. J. Amer. Chem. Soc. 133, 17807-17815 (2011).

59. Rodgers C. T., Norman S. A., Henbest K. B., Timmel C. R. \& Hore P. J. Determination of radical re-encounter probability distributions from magnetic field effects on reaction yields. J. Amer. Chem. Soc 129, 67466755 (2007).

60. Hoang N., et al. Human and Drosophila cryptochromes are light activated by flavin photoreduction in living cells. PLoS Biol. 6, e160 (2008).

61. Bouly J. P., et al. Cryptochrome blue light photoreceptors are activated through interconversion of flavin redox states. J. Biol. Chem. 282, 93839391 (2007). 


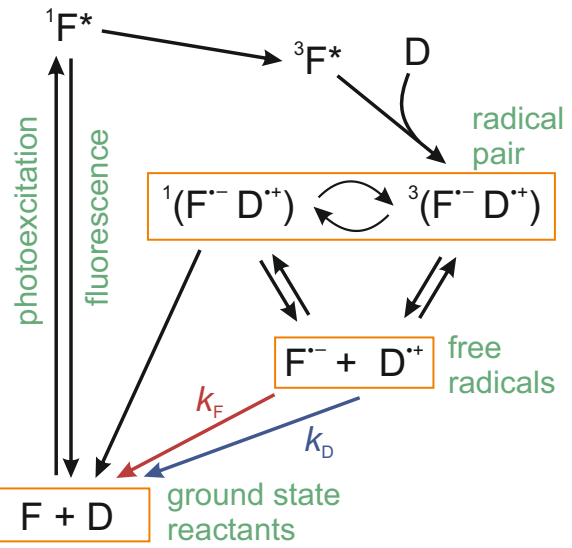




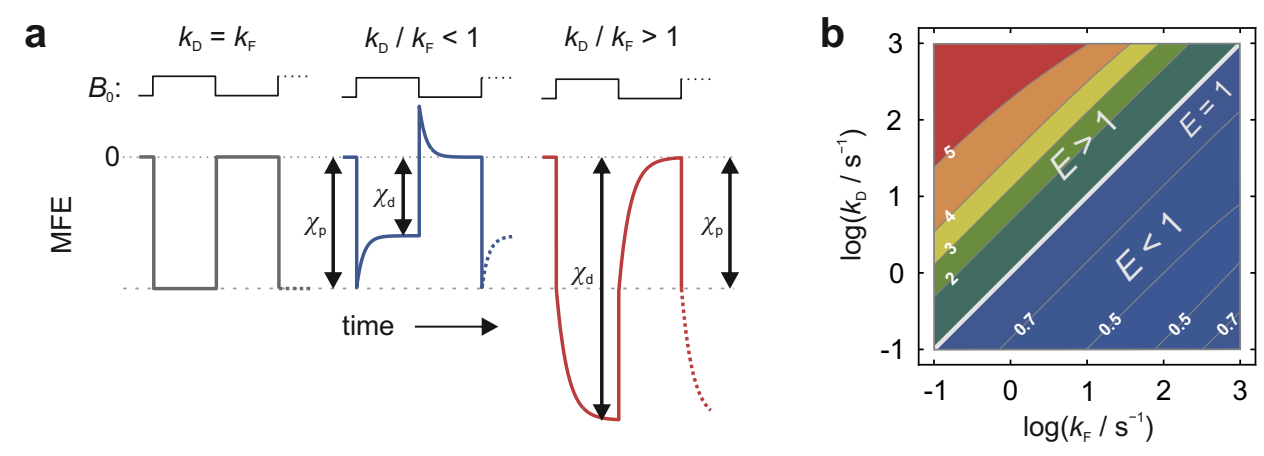




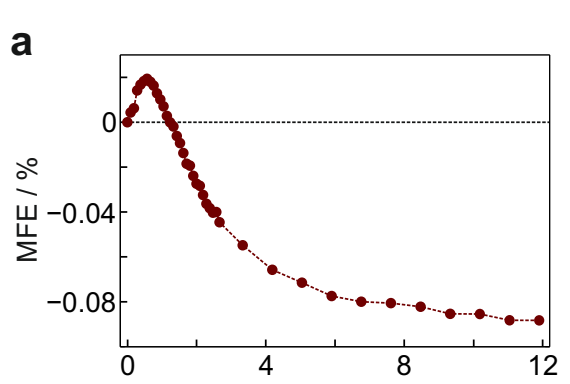

Magnetic field / $\mathrm{mT}$
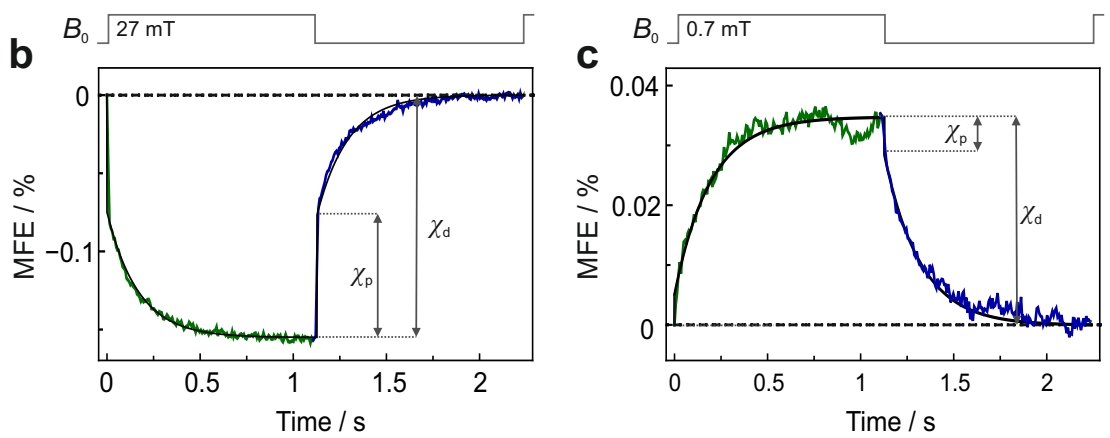


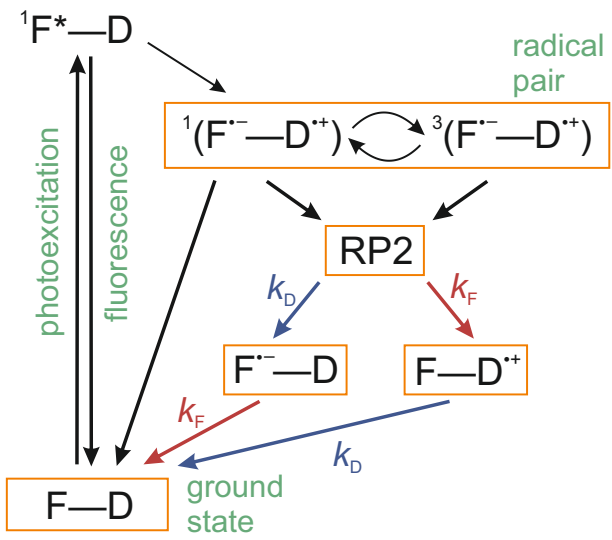


a

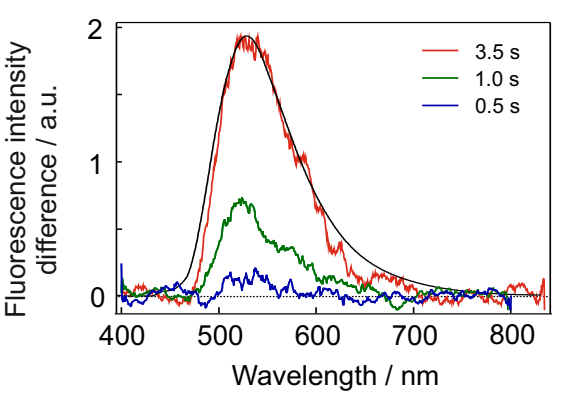

b $B_{0} \sqrt{27 \mathrm{mT}}$

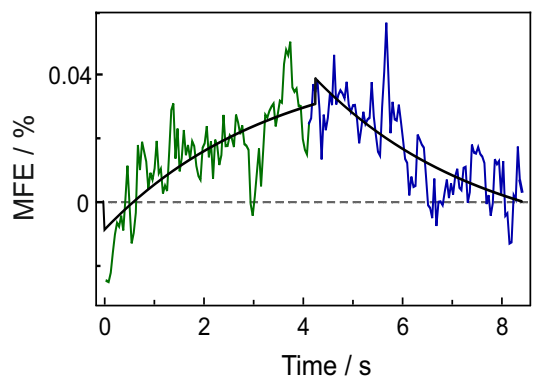

C $B_{0} \sqrt{27 \mathrm{mT}}$

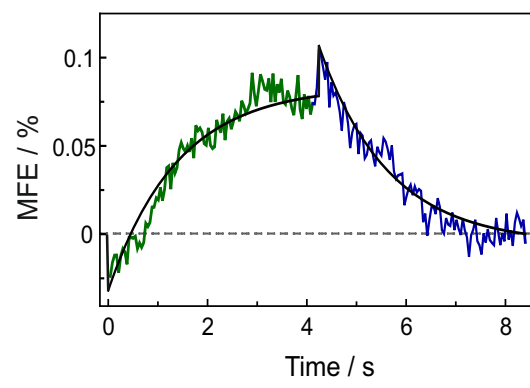


Chemical amplification of magnetic field effects relevant to avian magnetoreception

Daniel R. Kattnig, Emrys W. Evans, Victoire Déjean, Charlotte A. Dodson, Mark I. Wallace, Stuart R. Mackenzie, Christiane R. Timmel, P. J. Hore

\section{SUPPLEMENTARY INFORMATION}

\section{Contents}

A. Measurement of magnetic field effects by fluorescence 2

Spectrally resolved MFEs: Setup I 2

Time-resolved MFEs: Setup II 2

B. Measurement of magnetic field effects on intermolecular reactions of flavins 5

FMN/Lysozyme $\quad 5$

$\begin{array}{ll}\text { FMN/Tryptophan } & 7\end{array}$

C. Calculation of MFEs for inter- and intramolecular radical pairs 9

Intermolecular MFEs 9

Lower light intensities lead to larger enhancements 12

$\begin{array}{ll}\text { Intramolecular MFEs } & 14\end{array}$

D. Bare-bones model of MFEs for inter- and intramolecular radical pairs 17

$\begin{array}{ll}\text { Intermolecular MFEs } & 17\end{array}$

$\begin{array}{lr}\text { Intramolecular MFEs } & 19\end{array}$

$\begin{array}{ll}\text { E. Flavin photochemistry } & 20\end{array}$

Transient absorption $\quad 20$

Reactivity of flavin radicals $\quad 20$

$\begin{array}{ll}\text { F. Switched light intensity } & 23\end{array}$

Supplementary References $\quad 24$ 


\section{A. Measurement of magnetic field effects by fluorescence}

\section{Spectrally resolved MFEs: Setup I}

Magnetic field effects on fluorescence spectra were recorded essentially as described by Evans et al. ${ }^{1}$ Samples were positioned between Helmholtz coils which were used to generate static magnetic fields of up to $12 \mathrm{mT}$, with rise and fall times less than $3 \mathrm{~ms}$. A $405 \mathrm{~nm}$ CW diode laser (1Q1H350, $350 \mathrm{~mW}$; Power Technology) provided continuous illumination of the sample. The fluorescence was coupled to a spectrograph (SR303i; Andor) which dispersed the wavelength components onto a charge-coupled device (CCD) camera (DU90P-BV; Andor Newton). The fluorescence signal was integrated for a defined period (the camera 'exposure time') starting at a fixed 'delay time', $T$, after switching on the magnetic field. After recording the signal, the field was returned to zero ready for the next measurement. Most solutions were flowed at a rate of $c a .0 .25 \mathrm{~mL} \mathrm{~min}^{-1}$ through a sample cell (Starna Scientific, UV Quartz, 45/Q, $1 \mathrm{~mm}$ optical path length) so as to minimize photodegradation. Most of the experiments were conducted at room temperature and for magnetic fields in the range 0 to $12 \mathrm{mT}$, chosen in a randomized order. For AtCry1, the sample was not flowed and was cooled ( $282 \mathrm{~K}$ ) using a custom-made, chilled stage.

Experimental parameters for the results presented in the main text are given in Supplementary Table A1.

\begin{tabular}{llrrrr}
\hline Figure & Sample & $\begin{array}{r}\text { delay } \\
\text { time }\end{array}$ & $\begin{array}{r}\text { exposure } \\
\text { time }\end{array}$ & $\begin{array}{r}\text { excitation } \\
\text { power }\end{array}$ & averages \\
\hline 2c & FMN/lysozyme & $10,50 \mathrm{~ms}$ & $20 \mathrm{~ms}$ & $270 \mathrm{~mW}$ & 300 \\
& & $0.5 \mathrm{~s}$ & $20 \mathrm{~ms}$ & $270 \mathrm{~mW}$ & 100 \\
& & $2.0 \mathrm{~s}$ & $20 \mathrm{~ms}$ & $270 \mathrm{~mW}$ & 75 \\
& & $5.0 \mathrm{~s}$ & $20 \mathrm{~ms}$ & $270 \mathrm{~mW}$ & 35 \\
\hline 4a & FMN/ascorbic acid & $50 \mathrm{~ms}$ & $20 \mathrm{~ms}$ & $350 \mathrm{~mW}$ & 900 \\
\hline 6a & \multirow{2}{*}{ AtCry1 } & $0.5,1.0 \mathrm{~s}$ & $0.5 \mathrm{~s}$ & $1 \mathrm{~mW}$ & 300 \\
& & $3.5 \mathrm{~s}$ & $0.5 \mathrm{~s}$ & $1 \mathrm{~mW}$ & 80 \\
\hline
\end{tabular}

Supplementary Table A1. Setup I: Experimental parameters for the measurements shown in the main text.

\section{Time-resolved MFEs: Setup II}

Time-resolved measurements were carried out using an inverted fluorescence microscope (Ti-E; Nikon). Samples were held in a flow chamber constructed from a silicon rubber gasket (McMasterCarr) and two coverslips (Menzel; Germany) with an optical path length of $0.5 \mathrm{~mm}$ (see Ref. ${ }^{2}$ ). They were illuminated using the attenuated and expanded beam of a $470 \mathrm{~nm}$ diode laser (Stradus 473-80, $80 \mathrm{~mW}$; Vortran Laser Technology). The excitation light was focused at the back aperture of an oil immersion objective (CFI Apo TIRF 60X Oil, N.A. 1.49; Nikon) to provide trans-illumination of the sample. The power entering the back of the objective was varied between 0.10 and $3.7 \mathrm{~mW}$ resulting in power densities in the object plane between 0.020 and $0.74 \mu \mathrm{W} \mu \mathrm{m}^{-2}$ (based on the $1 / \mathrm{e}^{2}$ contours of the excitation intensity). The fluorescence emission was collected through the same objective, 
transmitted through a dichroic mirror (Brightline FF497-Di03; Semrock) and a bandpass filter (BrightLine 550/88; Semrock), and recorded using an electron-multiplying CCD camera (iXon+ DV860E; Andor Technology). Magnetic fields were generated using a solenoid wound around a $3 \mathrm{~mm}$ diameter ferrite core and fixed into a non-metallic mount placed $1 \mathrm{~mm}$ above the sample. Using a custom-built power supply, magnetic fields of up to $27 \mathrm{mT}$ could be generated with a rise time of less than $70 \mu \mathrm{s}$. Both the data acquisition and the magnetic field stepping were controlled by a digital delay pulse generator (model 9520; Quantum Composers). The excitation light was only admitted to the sample during the fluorescence measurement period using an optical shutter (SH05; Thorlabs) synchronized with the camera shutter. A description of the calibration of the magnetic field can be found in Ref. ${ }^{3}$.

Experimental parameters for the results presented in the main text are given in Supplementary Table A2.

For all samples except cryptochrome (Fig. 6, main text), the fluorescence was integrated for a period of $5 \mathrm{~ms}$ per camera frame (the camera 'exposure time') and the interval between frames was $7 \mathrm{~ms}$ (frame rate: $\sim 143 \mathrm{~Hz}$ ). Typically 16,000 frames were recorded with the magnetic field alternated between zero and a preset value every 80, 160, or 240 frames depending on the sample (see Supplementary Table A2 for details of individual measurements). The 'field on/off interval' is the time the magnetic field remains on or off before switching. For each run, a previously unexposed aliquot of the sample was admitted to the cell using a flow system. The flow was stopped while the fluorescence was measured.

For the cryptochrome experiments, $50 \mu \mathrm{L}$ samples were held between two coverslips separated by a polydimethylsiloxane spacer (Sylgard 184; Dow Corning). The sample was injected into the precooled cell placed on a Peltier stage (PT100-NIF; Linkam) at 268 K. 10,000 frames were recorded with an exposure time of $40 \mathrm{~ms}$ and frame interval of $42 \mathrm{~ms}$ with the field switched every 100 frames (i.e. 4.2 s). For each measurement at least 11 time-traces were accumulated, each time using a previously unexposed portion of the sample.

\begin{tabular}{|c|c|c|c|c|c|c|c|}
\hline Figure & Sample & $\begin{array}{r}\text { frame } \\
\text { interval }\end{array}$ & $\begin{array}{r}\text { exposure } \\
\text { time }\end{array}$ & frames & $\begin{array}{r}\text { field on/off } \\
\text { interval }\end{array}$ & $\begin{array}{r}\text { excitation } \\
\text { power }\end{array}$ & traces \\
\hline $2 a, b$ & FMN/lysozyme & $7 \mathrm{~ms}$ & $5 \mathrm{~ms}$ & 16,000 & $1.68 \mathrm{~s}$ & $0.24 \mathrm{~mW}$ & 16 \\
\hline $2 d, e$ & FMN/tryptophan & $7 \mathrm{~ms}$ & $5 \mathrm{~ms}$ & 16,000 & $0.56 \mathrm{~s}$ & $3.7 \mathrm{~mW}$ & 25 \\
\hline $2 f$ & $\begin{array}{l}\text { FMN/ tryptophan (+TCEP } \\
\text { or at } \mathrm{pH}=2.6 \text { ) }\end{array}$ & $7 \mathrm{~ms}$ & $5 \mathrm{~ms}$ & 16,000 & $1.12 \mathrm{~s}$ & $3.7 \mathrm{~mW}$ & 6,7 \\
\hline $4 b, c$ & FMN/ascorbic acid & $7 \mathrm{~ms}$ & $5 \mathrm{~ms}$ & 16,000 & $1.12 \mathrm{~s}$ & $3.7 \mathrm{~mW}$ & 35,63 \\
\hline $6 b, c$ & AtCry1 & $42 \mathrm{~ms}$ & $40 \mathrm{~ms}$ & 10,000 & $4.2 \mathrm{~s}$ & $0.1 \mathrm{~mW}$ & 15,11 \\
\hline
\end{tabular}

Supplementary Table A2. Setup II: Experimental parameters for the measurements shown in the main text.

The fluorescence intensity for each camera frame was obtained by 'robust averaging'. ${ }^{4}$ To determine the time-resolved MFE, the fluorescence time-profiles from individual measurements (as described above) were averaged, fitted to a multi-exponential function and used to calculate the MFEs for each field-on/field-off cycle. The first 5 cycles were discarded because of distortions resulting from 
the system adjusting to a new quasi-equilibrium during the initial phase of photoexcitation and because there was some difficulty fitting the average intensity due to the steep decay of the fluorescence at the beginning of the measurement. The time-resolved MFEs for the remaining on/off-cycles were averaged with the smallest and largest $5 \%$ of the values at every time point removed in order to guard against outliers and spikes. There were no statistically significant differences (at the $95 \%$ level) between the remaining time points and their means. In order to determine the prompt and the delayed MFEs, the time-resolved MFEs were fitted to exponential functions starting with the fourth time point. The MFEs extrapolated to $t=0$ and $t \rightarrow \infty$ are reported as $\chi_{\mathrm{p}}$ and $\chi_{\mathrm{d}}$, respectively. 


\section{B. Measurement of magnetic field effects on intermolecular reactions of flavins}

\section{FMN/Lysozyme}

Using setup I, fluorescence-detected MFE profiles were recorded for FMN/lysozyme mixtures at various delay times, $T$, between switching on the magnetic field and recording the fluorescence. A $10 \mu \mathrm{M}$ FMN/0.5 mM lysozyme sample was flowed at $0.25 \mathrm{~mL} \mathrm{~min}^{-1}$ with continuous photoexcitation by a $405 \mathrm{~nm}$ laser at $270 \mathrm{~mW}$. The fluorescence spectral profile showed good agreement with the $\mathrm{S}_{1} \rightarrow \mathrm{S}_{0}, \pi \pi^{*}$ transition of the isoalloxazine group of the flavin. A small decrease in fluorescence intensity was seen on application of an external magnetic field (12 mT). The inverted, scaled fluorescence difference signal for $T=0.01 \mathrm{~s}, \Delta I_{\mathrm{F}}\left(B_{0}\right)=I_{\mathrm{F}}\left(B_{0}\right)-I_{\mathrm{F}}(0)$, is shown as the black trace in Supplementary Fig. B1a; its sign is consistent with an initial triplet radical pair. The difference spectrum coincides perfectly with the fluorescence profile of fully oxidised FMN (red trace), suggesting a single dominant, magnetically sensitive fluorescent species (the excited singlet state of fully oxidised FMN). This conclusion is reinforced by the coincidence of the scaled difference signals obtained for different delay times between the field change and the start of fluorescence acquisition (Supplementary Fig. B1b).

a

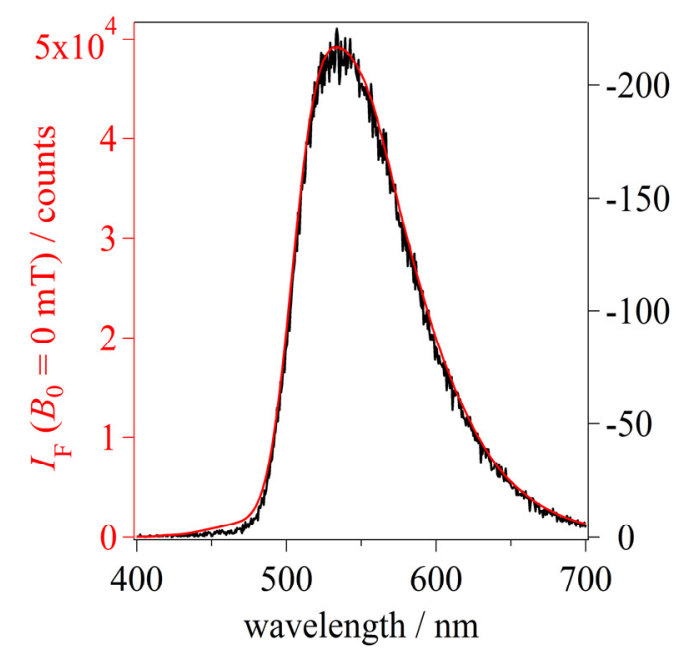

b

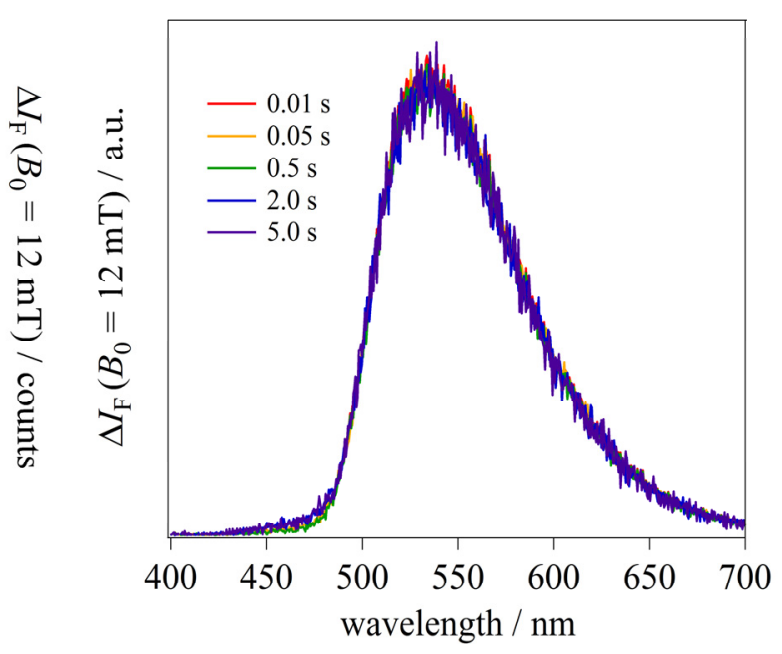

Supplementary Figure B1. a, Superimposed fluorescence intensity $I_{\mathrm{F}}\left(B_{0}=0\right)(\mathrm{red})$ and difference spectra $\Delta I_{\mathrm{F}}\left(B_{0}=12 \mathrm{mT}\right)$ (black) for $10 \mu \mathrm{M} \mathrm{FMN}+0.5 \mathrm{mM}$ lysozyme in water. Delay time, $T=0.01$ s. $\mathbf{b}$, Scaled difference spectra for the same system at different delay times between the field step and the start of fluorescence acquisition.

In Fig. 2c of the main text, an MFE amplification (averaged over 500-600 nm) was observed with increasing delay time $T$, whilst the $B_{1 / 2}$ (magnetic field strength corresponding to half of the maximum MFE) is unchanged (Supplementary Table B1). The invariance of $B_{1 / 2}$ is in keeping with our proposal that the enhanced MFEs stem from a single radical pair. 


\begin{tabular}{lccccc}
\hline delay $/ \mathrm{s}$ & 0.01 & 0.05 & 0.5 & 2.0 & 5.0 \\
\hline$B_{1 / 2} / \mathrm{mT}$ & $6.3 \pm 0.1$ & $6.5 \pm 0.2$ & $6.5 \pm 0.3$ & $6.5 \pm 0.2$ & $6.3 \pm 0.3$ \\
\hline
\end{tabular}

Supplementary Table B1. Values of $B_{1 / 2}$ determined from the data shown in Fig. 2c.

Setup II was used to investigate the time-dependence of the enhanced MFE. Fresh samples were continuously illuminated in the presence of a magnetic field stepped between $0 \mathrm{mT}$ and $27 \mathrm{mT}$ (rise time $<70 \mu \mathrm{s}$ ) recording the fluorescence continuously. The overall decrease in fluorescence intensity (Fig. 2a and d, main text) is due to the sample re-equilibrating to photoexcitation and the interplay between photobleaching and diffusion. Turning on the photoexcitation source rapidly depletes the flavin ground state as steady state concentrations for the excited states are reached. The photochemistry of flavins is not as perfectly cyclic as implied by the reaction scheme in Fig. 1 (main text). Photoexcitation also leads to degradation products such as lumichrome. ${ }^{5}$ Although lumichrome is also capable of forming photo-induced triplet-born radical pairs, no magnetically sensitive fluorescence from lumichrome was observable with $470 \mathrm{~nm}$ photoexcitation in setup II. Such photodegradation pathways tend to reduce the measured fluorescence intensity, although this is opposed by diffusion of fresh sample into the detection volume.

As mentioned in the main text, the time-resolved MFE is superimposed on the decaying trend of the fluorescence. An average MFE response to field switching was obtained following the subtraction of the decay by means of multi-exponential fits. No statistically significant difference was observed when comparing individual cycles to their means. Furthermore, the averaged MFE responses to field-on and field-off steps were identical (see Supplementary Fig. B2).

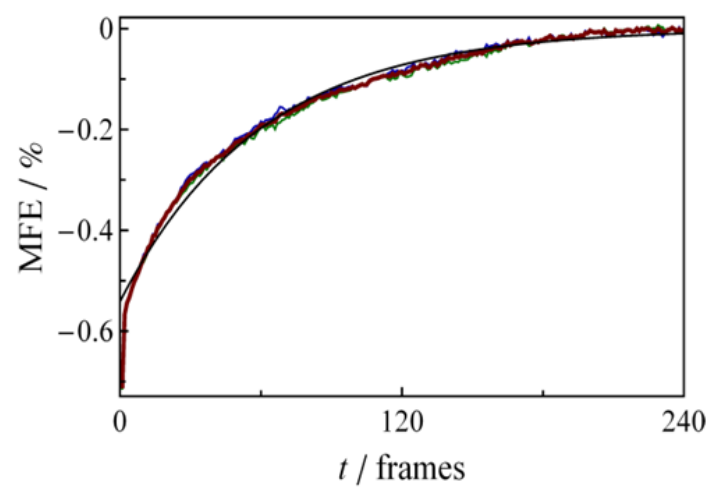

Supplementary Figure B2. Averaged MFEs for field-on and field-off steps. Green: response to switching field on. Blue: response to switching field off. Red: average of the two. Black:

exponential fit.

To obtain the enhancement factor, $E$, the initial (primary, $\chi_{\mathrm{p}}$ ) and final (delayed, $\chi_{\mathrm{d}}$ ) MFEs were determined. An exponential function was fitted to the slow component (see, e.g., Supplementary Fig. B2) to obtain a time constant $(\tau)$. Using such fits values of $\chi_{\mathrm{d}}$ could be obtained for reactions that did not reach a steady state during the field on/off periods. 
Increasing the solvent viscosity of the FMN/lysozyme solutions by the addition of glycerol $(20 \% \mathrm{v} / \mathrm{v})$ was found to boost the enhancement (Supplementary Fig. B3) from 2.32 to 3.50, as well as increasing the time constant from $\tau=0.47 \mathrm{~s}$ to $\tau=2.2 \mathrm{~s}$. While the ratio of the two termination rate constants $\left(k_{\mathrm{D}} / k_{\mathrm{F}}\right)$ determines $E$, it is their values which set the timescale. It seems likely that the increase in solvent viscosity affects the diffusion-controlled contributions to $k_{\mathrm{D}}$ and $k_{\mathrm{F}}$ and hence both $E$ and $\tau$.
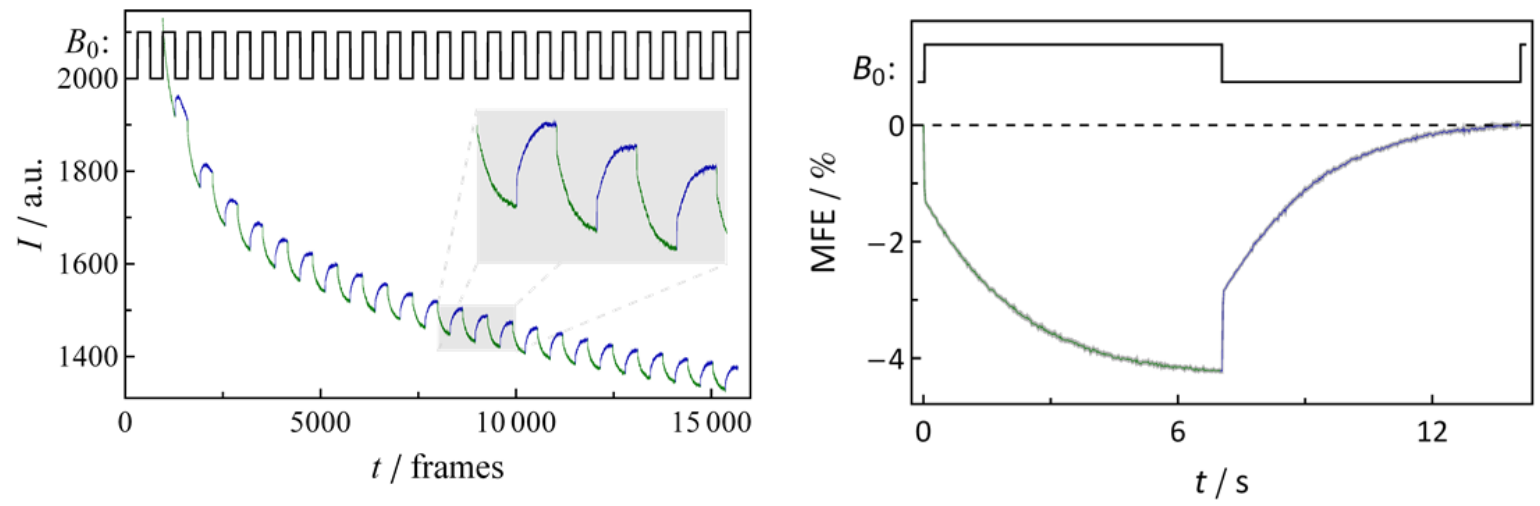

Supplementary Figure B3. Left: Fluorescence intensity profile for $10 \mu \mathrm{M} F M N+0.5 \mathrm{mM}$ lysozyme ( $20 \% \mathrm{v} / \mathrm{v}$ glycerol in water) in the presence of a square wave modulated magnetic field (0 to 27 $\mathrm{mT}$ ) using Setup II. Right: the averaged response to a single field step.

\section{FMN/Tryptophan}

The rate of disappearance of flavin radicals by disproportionation and reaction with molecular oxygen increases supra-linearly with their concentration as a result of the quadratic and autocatalytic nature, respectively, of the two processes. As expected and shown in Supplementary Figs $\mathrm{B} 4$ and $\mathrm{B} 5$, increasing either the FMN concentration or the laser pump power increases $k_{\mathrm{F}}$, decreases $k_{\mathrm{D}} / k_{\mathrm{F}}$ and results in smaller enhancement factors. 

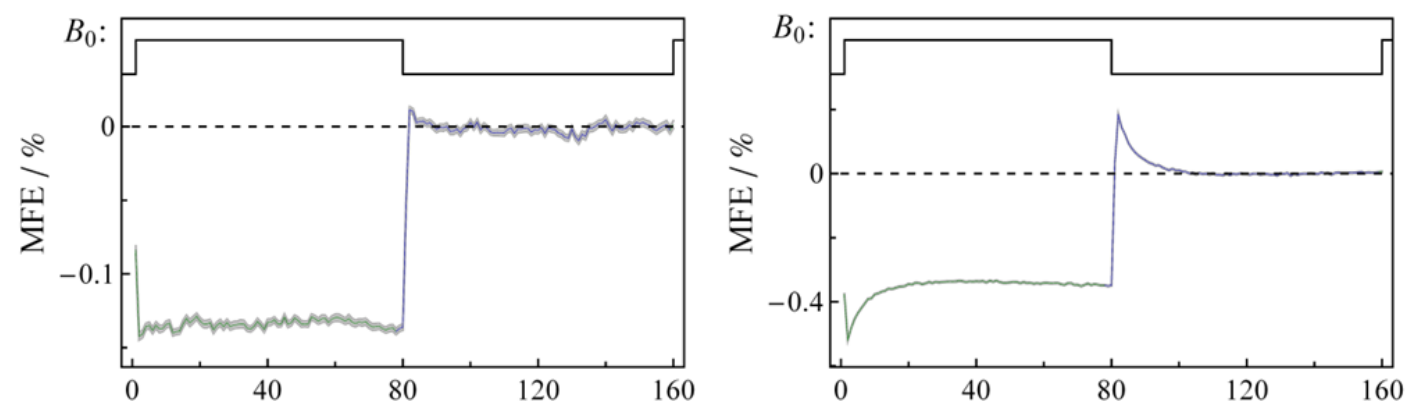

Supplementary Figure B4. Averaged MFE responses to a single field step (0-27 mT) for $10 \mu \mathrm{M}$ $\mathrm{FMN}+1.0 \mathrm{mM}$ tryptophan with varying laser pump power $(470 \mathrm{~nm})$ : left, $0.24 \mathrm{~mW}$ and right, $3.7 \mathrm{~mW}$.
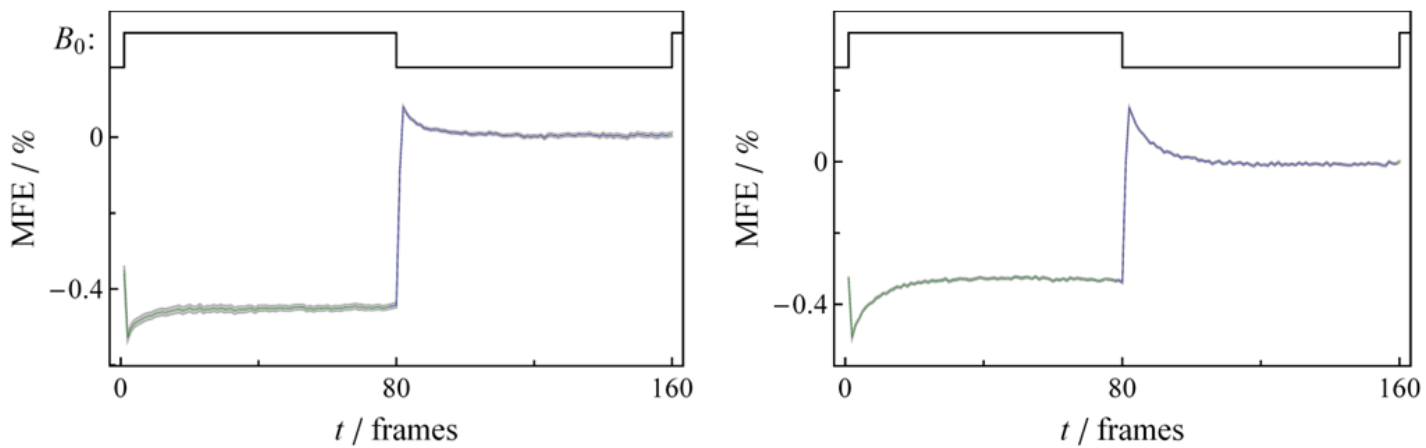

Supplementary Figure B5. Averaged MFE responses to a single field step (0-27 mT) for FMN + $1.0 \mathrm{mM}$ tryptophan for different flavin concentrations: left, $2.5 \mu \mathrm{M}$ and right, $10 \mu \mathrm{M}$. 


\section{Calculation of MFEs for inter- and intramolecular radical pairs}

\section{Intermolecular MFEs}

The reaction scheme in Supplementary Fig. $\mathrm{C} 1$ has been used to model the MFEs that arise in cyclic intermolecular radical pair reactions under conditions of continuous illumination and square-wave modulated magnetic fields. Using an approach analogous to that of Murakami et al. ${ }^{6}$, we have solved numerically the differential equations that describe the time-dependence of the system following step-wise changes in the intensity of an external magnetic field.

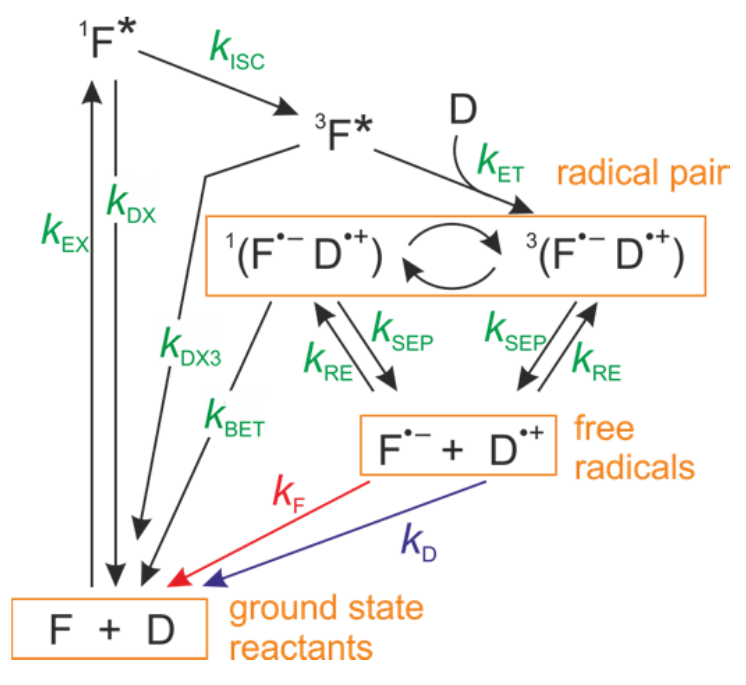

Supplementary Figure C1. Reaction scheme for intermolecular flavin $(F)+$ donor (D) reactions.

The magnetic sensitivity of the photocycle originates in field-dependent singlet-triplet interconversion of the singlet $(\mathrm{S})$ and triplet $\left(\mathrm{T}_{0}, \mathrm{~T}_{+}, \mathrm{T}_{-}\right)$spin states of the geminate radical pair (curly arrows in Supplementary Fig. $\mathrm{C} 1$ ). The field-independent rate equations for species other than the radical pair are:

$$
\begin{aligned}
& \frac{\mathrm{d}}{\mathrm{d} t}[\mathrm{~F}]=-k_{\mathrm{EX}}[\mathrm{F}]+k_{\mathrm{DX}}\left[{ }^{1} \mathrm{~F}^{*}\right]+k_{\mathrm{DX} 3}\left[{ }^{3} \mathrm{~F}^{*}\right]+k_{\mathrm{BET}}\left[\mathrm{S}_{0}\right]+k_{\mathrm{F}}\left[\mathrm{F}^{*-}\right] \\
& \frac{\mathrm{d}}{\mathrm{d} t}\left[{ }^{1} \mathrm{~F}^{*}\right]=-\left(k_{\mathrm{ISC}}+k_{\mathrm{DX}}\right)\left[{ }^{1} \mathrm{~F}^{*}\right]+k_{\mathrm{EX}}[\mathrm{F}] \\
& \frac{\mathrm{d}}{\mathrm{d} t}\left[{ }^{3} \mathrm{~F}^{*}\right]=-\left(k_{\mathrm{ET}}[\mathrm{D}]+k_{\mathrm{DX} 3}\right)\left[{ }^{3} \mathrm{~F}^{*}\right]+k_{\mathrm{ISC}}\left[{ }^{1} \mathrm{~F}^{*}\right] \\
& \frac{\mathrm{d}}{\mathrm{d} t}\left[\mathrm{~F}^{*}\right]=-\left(k_{\mathrm{RE}}\left[\mathrm{D}^{*+}\right]+k_{\mathrm{F}}\right)\left[\mathrm{F}^{*}\right]+k_{\mathrm{SEP}}\left([\mathrm{S}]+\left[\mathrm{T}_{0}\right]+\left[\mathrm{T}_{+}\right]+\left[\mathrm{T}_{-}\right]\right) \\
& \frac{\mathrm{d}}{\mathrm{d} t}[\mathrm{D}]=-k_{\mathrm{ET}}\left[{ }^{3} \mathrm{~F}^{*}\right][\mathrm{D}]+k_{\mathrm{BET}}[\mathrm{S}]+k_{\mathrm{D}}\left[\mathrm{D}^{\cdot+}\right] \\
& \frac{\mathrm{d}}{\mathrm{d} t}\left[\mathrm{D}^{*+}\right]=-\left(k_{\mathrm{RE}}\left[\mathrm{F}^{*-}\right]+k_{\mathrm{D}}\right)\left[\mathrm{D}^{*+}\right]+k_{\mathrm{SEP}}\left([\mathrm{S}]+\left[\mathrm{T}_{0}\right]+\left[\mathrm{T}_{+}\right]+\left[\mathrm{T}_{-}\right]\right) .
\end{aligned}
$$

Note that the first terms on the right hand side of the 4th and 6th equations (rate constant $k_{\mathrm{RE}}$ ) do not change the total flavin or donor radical concentrations, merely whether these species are 
present in the radical pair or as free radicals. The concentrations of the latter greatly exceed those of the former.

In the absence of a magnetic field, all four spin states of the radical pair can interconvert with each other via the hyperfine interactions with the rate constant $k_{\text {HFM }}$ :

$$
\begin{aligned}
& \frac{\mathrm{d}}{\mathrm{d} t}[\mathrm{~S}]=-\left(3 k_{\mathrm{HFM}}+k_{\mathrm{BET}}+k_{\mathrm{SEP}}\right)[\mathrm{S}]+k_{\mathrm{HFM}}\left(\left[\mathrm{T}_{0}\right]+\left[\mathrm{T}_{+}\right]+\left[\mathrm{T}_{-}\right]\right)+\frac{1}{4} k_{\mathrm{RE}}\left[\mathrm{F}^{--}\right]\left[\mathrm{D}^{++}\right] \\
& \frac{\mathrm{d}}{\mathrm{d} t}\left[\mathrm{~T}_{0}\right]=-\left(3 k_{\mathrm{HFM}}+k_{\mathrm{SEP}}\right)\left[\mathrm{T}_{0}\right]+k_{\mathrm{HFM}}\left([\mathrm{S}]+\left[\mathrm{T}_{+}\right]+\left[\mathrm{T}_{-}\right]\right)+\frac{1}{4} k_{\mathrm{RE}}\left[\mathrm{F}^{--}\right]\left[\mathrm{D}^{++}\right]+\frac{1}{3} k_{\mathrm{ET}}\left[{ }^{3} \mathrm{~F}^{*}\right][\mathrm{D}] \\
& \left.\frac{\mathrm{d}}{\mathrm{d} t}\left[\mathrm{~T}_{+}\right]=-\left(2 k_{\mathrm{HFM}}+k_{\mathrm{SEP}}\right)\left[\mathrm{T}_{+}\right]+k_{\mathrm{HFM}}\left([\mathrm{S}]+\left[\mathrm{T}_{0}\right]\right)+\frac{1}{4} k_{\mathrm{RE}}\left[\mathrm{F}^{-*}\right]\left[\mathrm{D}^{++}\right]+\frac{1}{3} k_{\mathrm{ET}}{ }^{3} \mathrm{~F}^{*}\right][\mathrm{D}] \\
& \frac{\mathrm{d}}{\mathrm{d} t}\left[\mathrm{~T}_{-}\right]=-\left(2 k_{\mathrm{HFM}}+k_{\mathrm{SEP}}\right)\left[\mathrm{T}_{-}\right]+k_{\mathrm{HFM}}\left([\mathrm{S}]+\left[\mathrm{T}_{0}\right]\right)+\frac{1}{4} k_{\mathrm{RE}}\left[\mathrm{F}^{-*}\right]\left[\mathrm{D}^{\cdot+}\right]+\frac{1}{3} k_{\mathrm{ET}}\left[{ }^{3} \mathrm{~F}^{*}\right][\mathrm{D}] .
\end{aligned}
$$

At high magnetic field, the $T_{+}$and $T_{-}$states become energetically separated from $S$ and $T_{0}$ by the Zeeman interaction. This disables $\mathrm{ST}_{ \pm}$interconversion such that the rate equations for the geminate radical pair become:

$$
\begin{aligned}
\frac{\mathrm{d}}{\mathrm{d} t}[\mathrm{~S}] & =-\left(k_{\mathrm{HFM}}+2 k_{\mathrm{RM}}+k_{\mathrm{BET}}+k_{\mathrm{SEP}}\right)[\mathrm{S}]+k_{\mathrm{HFM}}\left[\mathrm{T}_{0}\right]+k_{\mathrm{RM}}\left(\left[\mathrm{T}_{+}\right]+\left[\mathrm{T}_{-}\right]\right)+\frac{1}{4} k_{\mathrm{RE}}\left[\mathrm{F}^{*}\right]\left[\mathrm{D}^{+}\right] \\
\frac{\mathrm{d}}{\mathrm{d} t}\left[\mathrm{~T}_{0}\right] & =-\left(k_{\mathrm{HFM}}+2 k_{\mathrm{RM}}+k_{\mathrm{SEP}}\right)\left[\mathrm{T}_{0}\right]+k_{\mathrm{HFM}}[\mathrm{S}]+k_{\mathrm{RM}}\left(\left[\mathrm{T}_{+}\right]+\left[\mathrm{T}_{-}\right]\right)+\frac{1}{4} k_{\mathrm{RE}}\left[\mathrm{F}^{*}\right]\left[\mathrm{D}^{+}\right]+\frac{1}{3} k_{\mathrm{ET}}\left[{ }^{3} \mathrm{~F}^{*}\right][\mathrm{D}] \\
\frac{\mathrm{d}}{\mathrm{d} t}\left[\mathrm{~T}_{+}\right] & =-\left(2 k_{\mathrm{RM}}+k_{\mathrm{SEP}}\right)\left[\mathrm{T}_{+}\right]+k_{\mathrm{RM}}\left([\mathrm{S}]+\left[\mathrm{T}_{0}\right]\right)+\frac{1}{4} k_{\mathrm{RE}}\left[\mathrm{F}^{-}\right]\left[\mathrm{D}^{++}\right]+\frac{1}{3} k_{\mathrm{ET}}\left[{ }^{3} \mathrm{~F}^{*}\right][\mathrm{D}] \\
\frac{\mathrm{d}}{\mathrm{d} t}\left[\mathrm{~T}_{-}\right] & =-\left(2 k_{\mathrm{RM}}+k_{\mathrm{SEP}}\right)\left[\mathrm{T}_{-}\right]+k_{\mathrm{RM}}\left([\mathrm{S}]+\left[\mathrm{T}_{0}\right]\right)+\frac{1}{4} k_{\mathrm{RE}}\left[\mathrm{F}^{-*}\right]\left[\mathrm{D}^{++}\right]+\frac{1}{3} k_{\mathrm{ET}}\left[{ }^{3} \mathrm{~F}^{*}\right][\mathrm{D}]
\end{aligned}
$$

$k_{\mathrm{RM}}$ is the rate constant for incoherent spin mixing resulting from spin relaxation processes. Supplementary Fig. $\mathrm{C} 2$ indicates the pathways described by the rate constants $k_{\mathrm{HFM}}$ and $k_{\mathrm{RM}}$.

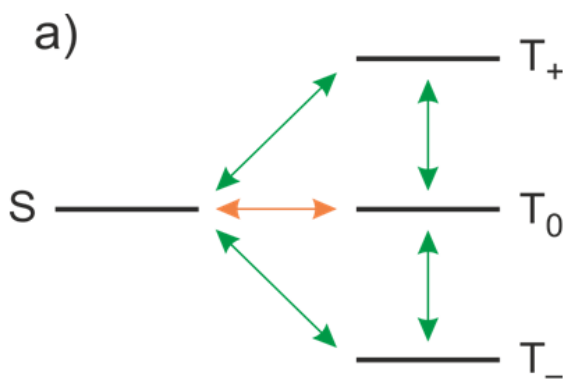

b)

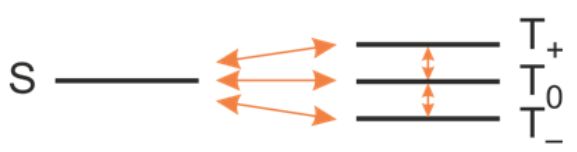

Supplementary Figure C2. Hyperfine mixing (orange arrows, rate constant $k_{\text {HFM }}$ ) and spin relaxation (green arrows, rate constant $k_{\mathrm{RM}}$ ) at $\mathbf{a}$ high field and $\mathbf{b}$ low field. 
MATLAB's $15 \mathrm{~s}$ ordinary differential equation solver was used to calculate the change in the concentration of ground state flavin when the applied magnetic field was switched on and off under conditions of continuous photoexcitation. The fluorescence intensity is directly proportional to [F].

Calculations were performed using the rate constants in Supplementary Table C1. As shown in Fig. 3a (main text), this yielded: (i) step-wise behaviour (enhancement factor, $E=\chi_{\mathrm{e}} / \chi_{\mathrm{d}}=1$ ) in the absence of termination channels $\left(k_{\mathrm{F}}=k_{\mathrm{D}}=0\right)$; (ii) $E>1$ for $k_{\mathrm{D}} / k_{\mathrm{F}}>1$; (iii) $E<1$ for $k_{\mathrm{D}} / k_{\mathrm{F}}<1$. Similar calculations were used to obtain the contour plot in Fig. $3 b$ (main text) showing the dependence of $E$ on $k_{\mathrm{D}} / k_{\mathrm{F}}$ for $k_{\mathrm{D}}$ and $k_{\mathrm{F}}$ in the range $10^{-1}-10^{3} \mathrm{~s}^{-1}$. The simulations used to obtain values of $k_{\mathrm{D}}$ and $k_{\mathrm{F}}$ from the data in Figs $2 b$, e and f, $4 b$ and $c$, and $6 b$ and $c$ were barely affected by the rates of the other reaction steps (Supplementary Table C1) which, for the small values of $k_{\mathrm{D}}$ and $k_{\mathrm{F}}$ relevant here, determine solely the prompt MFE.

In determining $E$, the final MFE response $\left(\chi_{\mathrm{d}}\right)$ was obtained from the steady state solution to Equations (1)-(3). The initial MFE $\left(\chi_{\mathrm{p}}\right)$ was defined at the moment of instantaneous magnetic field switching and was determined from the exponential fit to the enhanced MFE.

\begin{tabular}{|c|c|c|}
\hline Rate constant & Value & Comment \\
\hline$k_{\mathrm{EX}}$ & $1.36 \times 10^{4} \mathrm{~s}^{-1}$ & $\begin{array}{l}\text { Photoexcitation is assumed to follow first order kinetics in the weak } \\
\text { absorbance limit of the Beer-Lambert law. }\end{array}$ \\
\hline$k_{\mathrm{DX}}$ & $1.09 \times 10^{8} \mathrm{~s}^{-1}$ & $\begin{array}{l}\text { Decay of the flavin excited singlet state }\left({ }^{1} \mathrm{~F}^{*}\right) \text { by processes other than } \\
\text { intersystem-crossing. }\end{array}$ \\
\hline$k_{\mathrm{ISC}}$ & $1.09 \times 10^{8} \mathrm{~s}^{-1}$ & Intersystem crossing from ${ }^{1} \mathrm{~F}^{*}$ to ${ }^{3} \mathrm{~F}^{* 7,8}$. \\
\hline$k_{\mathrm{ET}}$ & $1.2 \times 10^{9} \mathrm{M}^{-1} \mathrm{~s}^{-1}$ & $\begin{array}{l}\text { Reaction of the flavin excited triplet state }\left({ }^{3} \mathrm{~F}^{*}\right) \text { with an electron donor, D. } \\
\text { The numerical value was derived from Stern-Volmer type experiments on } \\
\text { flavin mononucleotide (FMN) + tryptophan reactions using transient } \\
\text { absorption spectroscopy (see Section C). }\end{array}$ \\
\hline$k_{\mathrm{DX} 3}$ & $3.85 \times 10^{5} \mathrm{~s}^{-1}$ & $\begin{array}{l}\text { Decay of }{ }^{3} \mathrm{~F}^{*} \text { by processes other than quenching by } \mathrm{D} \text {. The numerical value } \\
\text { was taken from transient absorption experiments on FMN (see Section C). }\end{array}$ \\
\hline$k_{\mathrm{SEP}}$ & $2 \times 10^{8} \mathrm{~s}^{-1}$ & $\begin{array}{l}\text { Separation of geminate radical pair components to form free radicals. The } \\
\text { value is based on a typical geminate radical pair lifetime of } 100 \mathrm{~ns} \text { in } \\
\text { aqueous solution. }\end{array}$ \\
\hline$k_{\text {HFM }}$ & $8 \times 10^{7} \mathrm{~s}^{-1}$ & $\begin{array}{l}\text { Coherent singlet-triplet interconversion of the spin states of the geminate } \\
\text { radical pair by the hyperfine mechanism as determined by Murakami et al. }\end{array}$ \\
\hline$k_{\mathrm{RM}}$ & $2 \times 10^{6} \mathrm{~s}^{-1}$ & $\begin{array}{l}\text { Incoherent singlet-triplet interconversion of the spin states of the } \\
\text { geminate radical pair by the relaxation mechanism as determined by } \\
\text { Murakami et al. }{ }^{6}\end{array}$ \\
\hline$k_{\mathrm{RE}}$ & $1.87 \times 10^{10} \mathrm{M}^{-1} \mathrm{~s}^{-1}$ & $\begin{array}{l}\text { Re-encounter of free radicals to form geminate radical pair. The value is } \\
\text { obtained from the Smoluchowski equation (with an effective encounter } \\
\text { distance, } R^{*}=20 \AA \text { ) and the Stokes-Einstein relation }(T=295 \mathrm{~K} \text {; species } \\
\text { radius, } R=3.5 \AA \text {; solvent viscosity }=1 \times 10^{-2} \mathrm{~kg} \mathrm{~m}^{-1} \mathrm{~s}^{-1} \text { ). }\end{array}$ \\
\hline
\end{tabular}




\begin{tabular}{|l|l|l|}
\hline$k_{\mathrm{BET}}$ & $1.0 \times 10^{8} \mathrm{~s}^{-1}$ & $\begin{array}{l}\text { Spin-selective reverse electron transfer of the radical pair to form the } \\
\text { ground state reactants. }\end{array}$ \\
\hline
\end{tabular}

Supplementary Table C1. Values of the rate constants used in calculations of amplified MFEs for the intermolecular flavin photocycle in Supplementary Fig. C1.

\section{Lower light intensities lead to larger enhancements}

Fig. $3 \mathrm{~b}$ (main text) shows the dependence of $E$ on $k_{\mathrm{D}}$ and $k_{\mathrm{F}}$ for a typical intermolecular flavincontaining radical pair. Those calculations used the values of the rate constants given in Supplementary Table C1; the resultant density plot is also given below (Supplementary Fig. C3a) together with plots of the constituent delayed $\left(\chi_{\mathrm{d}}\right)$ and prompt $\left(\chi_{\mathrm{p}}\right)$ MFEs (Supplementary Figs. C3b and C3c, respectively).
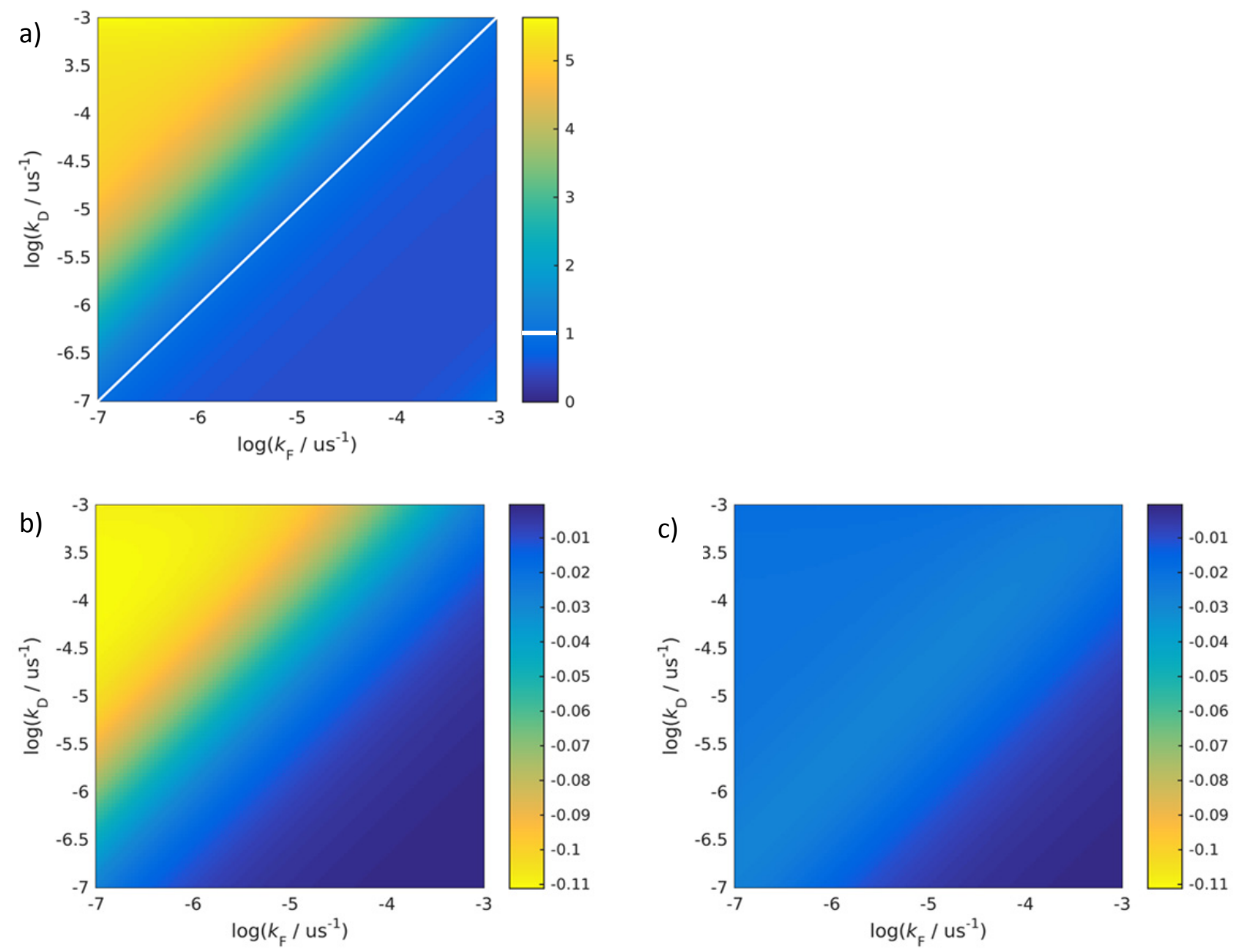

Supplementary Figure C3. Intermolecular radical pair. Density plots of: a, the amplification factor, $E ; \mathbf{b}$, the delayed MFE, $\chi_{\mathrm{d}} ; \mathbf{c}$ the prompt MFE, $\chi_{\mathrm{p}}$, as a function of the radical termination rate constants $k_{\mathrm{F}}$ and $k_{\mathrm{D}}$. The simulations were performed using the rate constant values in Supplementary Table C1 with $k_{\mathrm{EX}}=1.36 \times 10^{4} \mathrm{~s}^{-1}$. 
As mentioned in the main text, larger MFE amplifications can be realized with lower light intensities. The calculated behaviour for a sample subjected to $20 \%$ of the photon flux (i.e. $k_{\mathrm{EX}}=2.72 \times 10^{3} \mathrm{~s}^{-1}$ ) is shown in Supplementary Fig. C4. Enhancement factors of up to 24 are observed in the range of $k_{\mathrm{F}}$ and $k_{\mathrm{D}}$ values covered.
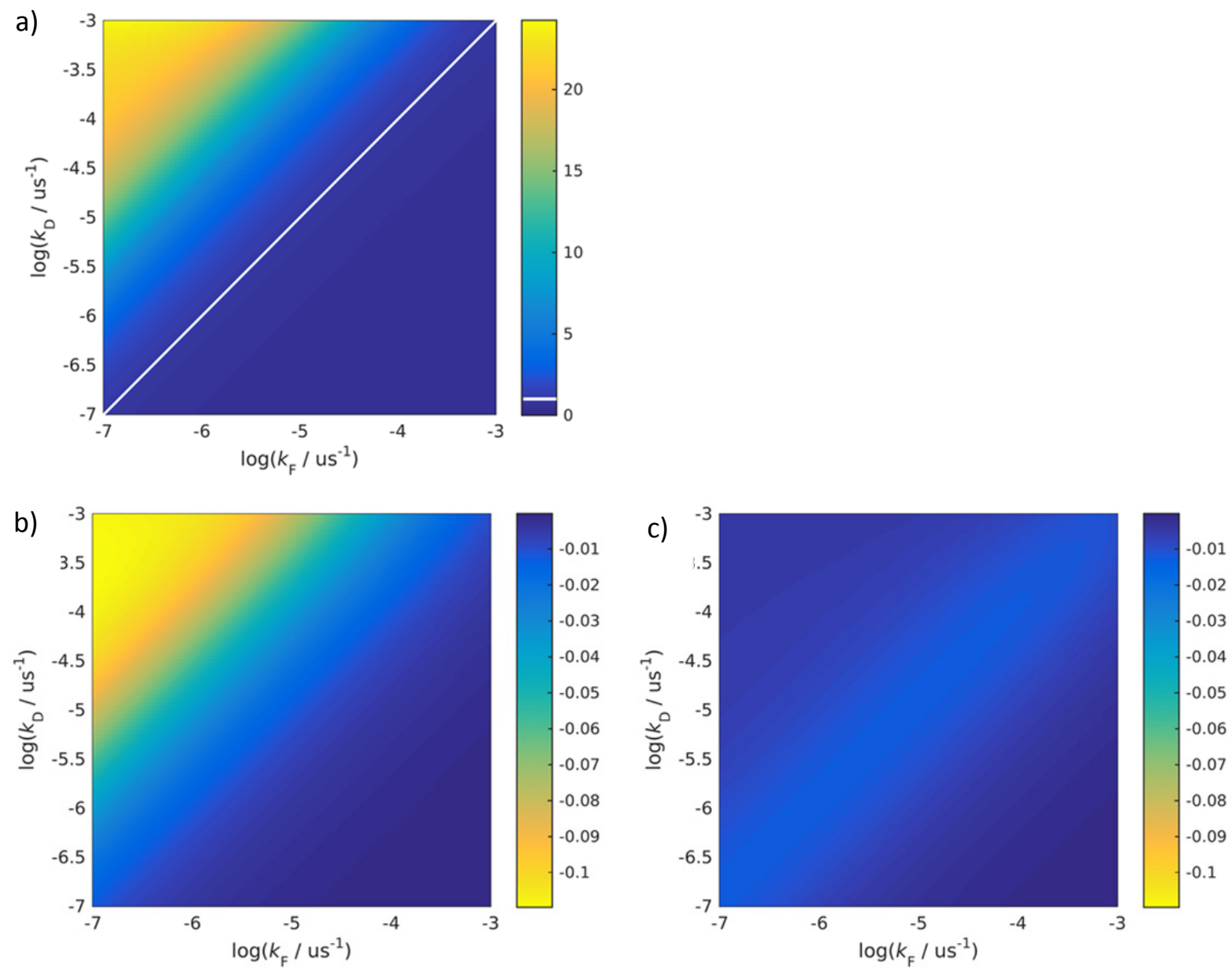

Supplementary Figure C4. Intermolecular radical pair. Density plots of: a, the amplification factor, $E ; \mathbf{b}$, the delayed MFE, $\chi_{\mathrm{d}} ; \mathbf{c}$, the prompt MFE, $\chi_{\mathrm{p}}$, as a function of the radical termination rate constants $k_{\mathrm{F}}$ and $k_{\mathrm{D}}$. The simulations were performed using the rate constant values in Supplementary Table C1 except for $k_{\mathrm{EX}}=2.72 \times 10^{3} \mathrm{~s}^{-1}$. 


\section{Intramolecular MFEs}

Analogous calculations of EMFEs were conducted for an intramolecular photocycle based on cryptochrome (Supplementary Fig. C5 and Supplementary Table C2). The corresponding density plots are shown in Supplementary Fig. C6-C8.

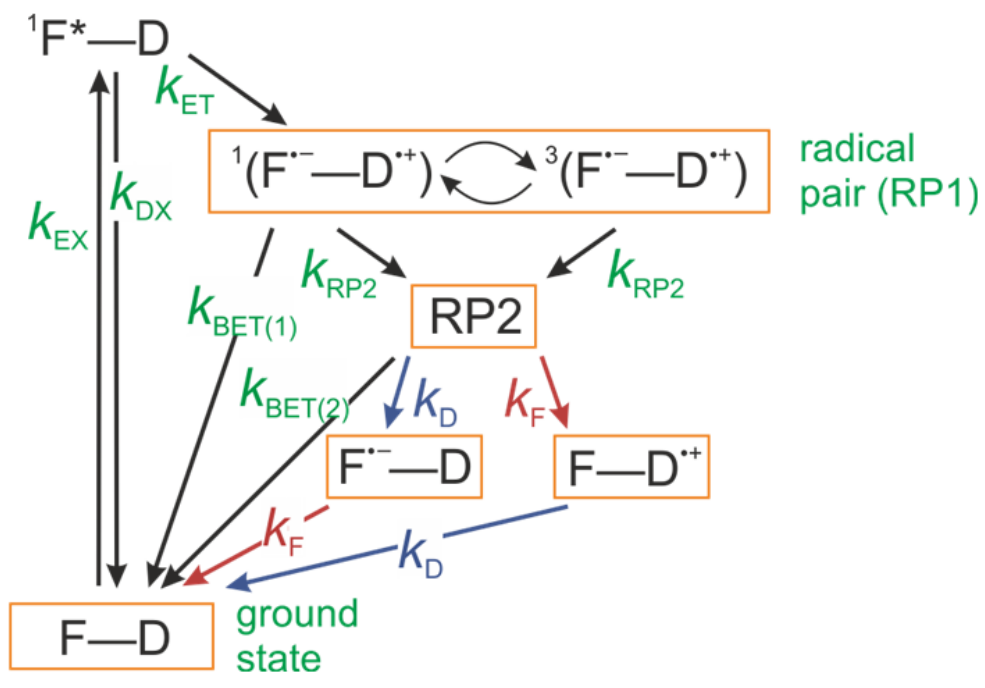

Supplementary Figure $\mathbf{C 5}$. Reaction scheme for an intramolecular radical pair reaction modelled on a typical cryptochrome photocycle.

\begin{tabular}{|c|c|c|}
\hline Rate constant & Value & Comment \\
\hline$k_{\mathrm{EX}}$ & $1 \times 10^{3} \mathrm{~s}^{-1}$ & $\begin{array}{l}\text { Photoexcitation is assumed to follow first order kinetics in the weak } \\
\text { absorbance limit of the Beer-Lambert law. }\end{array}$ \\
\hline$k_{\mathrm{DX}}$ & $1.09 \times 10^{8} \mathrm{~s}^{-1}$ & $\begin{array}{l}\text { Decay of the flavin excited singlet state }\left({ }^{1} \mathrm{~F}^{*}\right) \text { by processes other than } \\
\text { electron transfer. Value from Supplementary Table } \mathrm{C} 1 \text {. }\end{array}$ \\
\hline$k_{\mathrm{ET}}$ & $1 \times 10^{10} \mathrm{~s}^{-1}$ & $\begin{array}{l}\text { Efficient electron transfer between donor and flavin singlet excited state, } \\
{ }^{1} \mathrm{~F}^{*} \text {, to form RP1 in the singlet state. }\end{array}$ \\
\hline$k_{\mathrm{RP} 2}$ & $2 \times 10^{6} \mathrm{~s}^{-1}$ & Proton transfer processes to form RP2 from RP1 ${ }^{9}$. \\
\hline$k_{\mathrm{HFM}}$ & $8 \times 10^{7} s^{-1}$ & $\begin{array}{l}\text { Coherent singlet-triplet interconversion of the spin states of the geminate } \\
\text { radical pair by the hyperfine mechanism. Value from Supplementary Table } \\
\text { C1. }\end{array}$ \\
\hline$k_{\mathrm{RM}}$ & $2 \times 10^{6} \mathrm{~s}^{-1}$ & $\begin{array}{l}\text { Incoherent singlet-triplet interconversion of the spin states of the } \\
\text { geminate radical pair by the relaxation mechanism. Value from } \\
\text { Supplementary Table C1. }\end{array}$ \\
\hline$k_{\mathrm{BET}(1)}$ & $2 \times 10^{6} \mathrm{~s}^{-1}$ & Reverse electron transfer of RP1 to form the ground state species ${ }^{9}$. \\
\hline$k_{\mathrm{BET}(2)}$ & $5 \times 10^{4} s^{-1}$ & $\begin{array}{l}\text { Reverse electron transfer of RP2 to form the ground state. Estimated from } \\
\text { Ref. }\end{array}$ \\
\hline
\end{tabular}

Supplementary Table C2. Values of the rate constants used in calculations of amplified MFEs for the intramolecular flavin photocycle in Supplementary Fig. C5. 
Supplementary Fig. C6 shows the relative changes in the total concentration of the form of the protein containing the flavin radical [i.e. RP2 plus $\left(\mathrm{F}^{\circ-} \mathrm{D}\right)$ ] while Supplementary Fig. $\mathrm{C} 7$ shows the corresponding absolute changes. Supplementary Fig. C8 is a repeat of Supplementary Fig. C7 with $k_{\mathrm{Ex}}$, the rate constant for photoexcitation, reduced by a factor of 10 to $1 \times 10^{2} \mathrm{~s}^{-1}$. Note that Supplementary Fig. C7 is identical to Figs $6 \mathrm{~d}$ and e in the main text and is reproduced here for comparison with Supplementary Figs. C6 and C8.

Prompt relative MFE

Steady state relative MFE
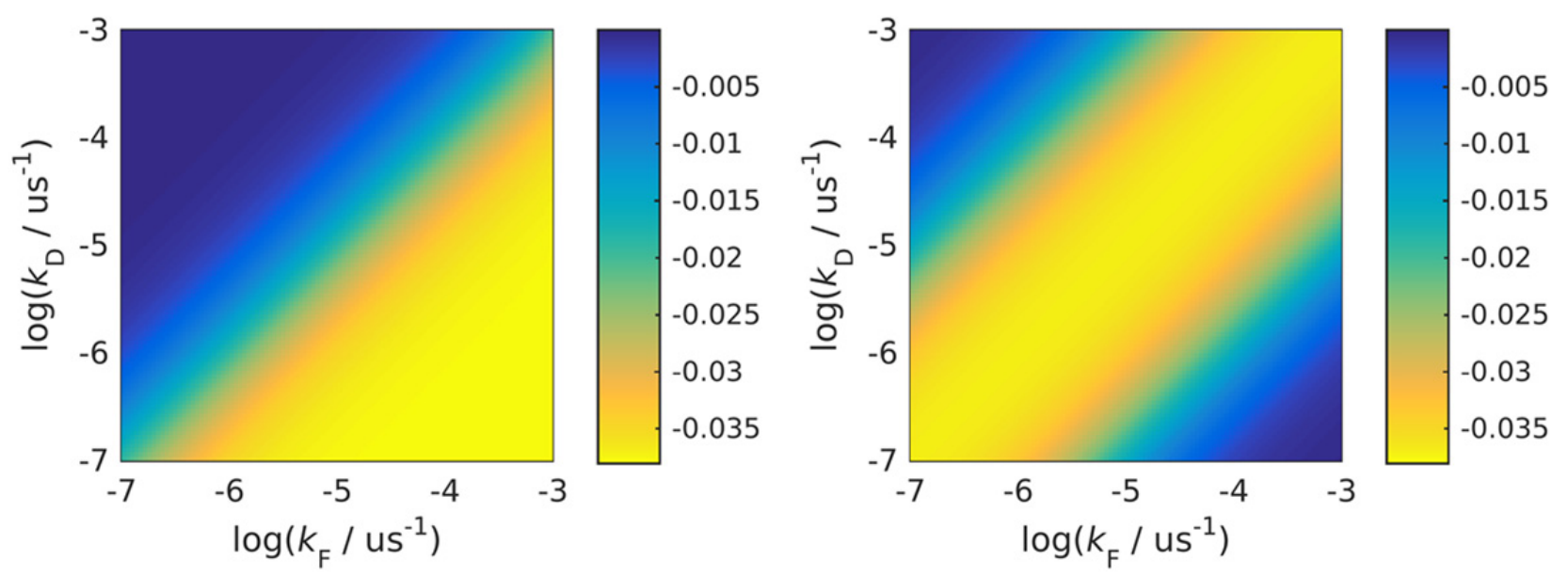

Supplementary Figure C6. Density plot of the relative changes in the total concentration of the flavin radical form of cryptochrome as a function of the radical termination rate constants $k_{\mathrm{F}}$ and $k_{\mathrm{D}}$. The values of the parameters are given Supplementary Table C2.

The maximum steady state relative MFE (Supplementary Fig. C6) is found for $k_{\mathrm{F}} \approx k_{\mathrm{D}}$ and does not exceed the maximum prompt effect (attained for $k_{\mathrm{F}} \gg k_{\mathrm{D}}$ ). While this does demonstrate that slow termination reactions play a role, their impact on the operation of cryptochrome as a magnetic sensor appears to be minor.

The picture is quite different for the corresponding absolute changes (Supplementary Fig. C7). The largest delayed effects occur when $k_{\mathrm{D}} \gg k_{\mathrm{F}}$ and they are substantially larger than the prompt effect for any values of $k_{\mathrm{D}}$ and $k_{\mathrm{F}}$.

Furthermore, when the light intensity is reduced (Supplementary Fig. C8), a larger ratio $k_{\mathrm{D}} / k_{\mathrm{F}}$ is necessary to achieve a significant enhancement (see Supplementary Information). That is, the lower the light intensity the more important is the enhancement phenomenon. 

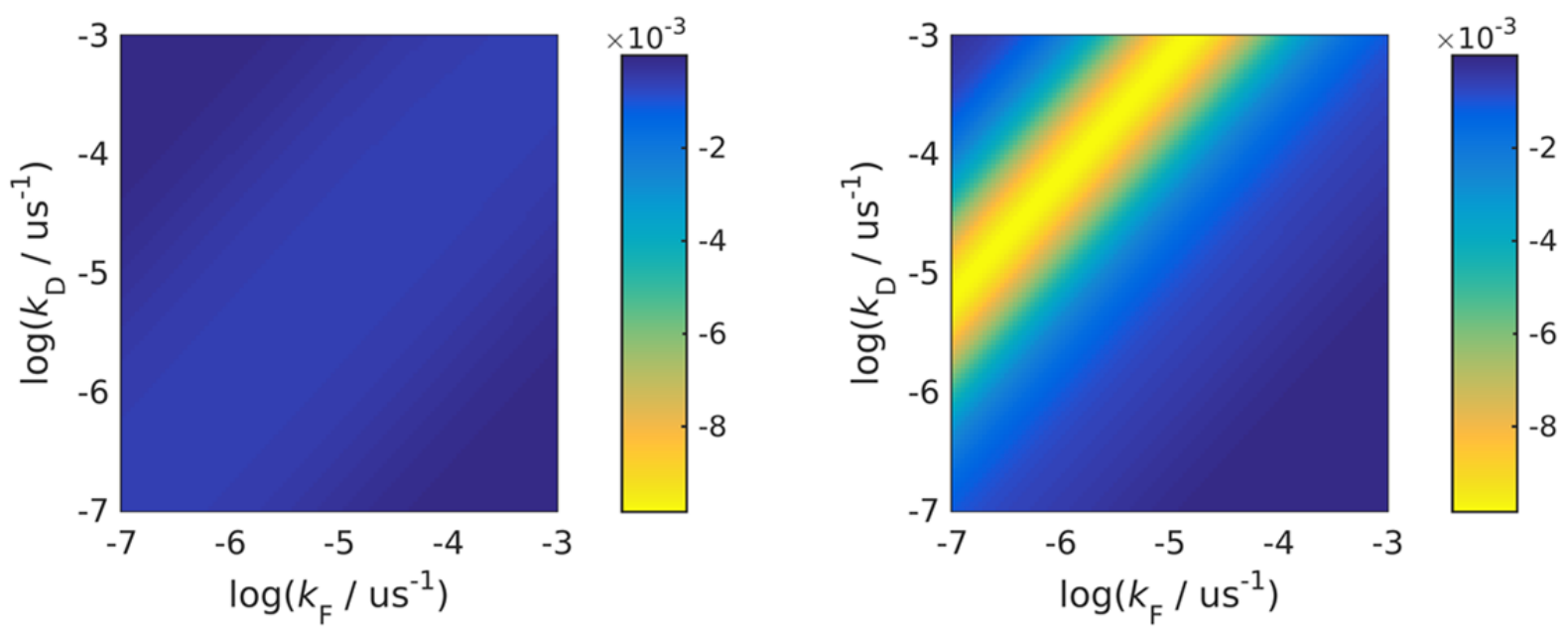

Supplementary Figure C7. Density plot of the absolute changes in the total concentration of the flavin radical form of cryptochrome as a function of the radical termination rate constants $k_{\mathrm{F}}$ and $k_{\mathrm{D}}$. The values of the parameters are given Supplementary Table C2.

Prompt absolute MFE

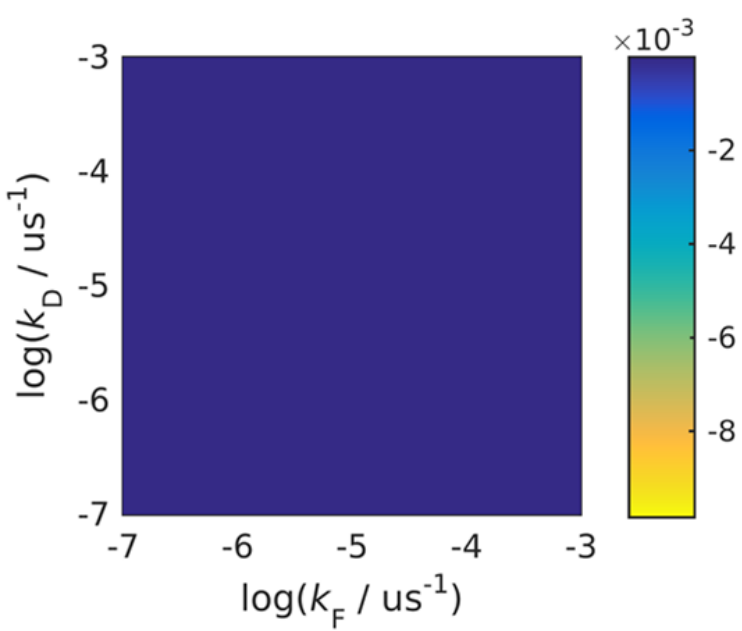

Steady state absolute MFE

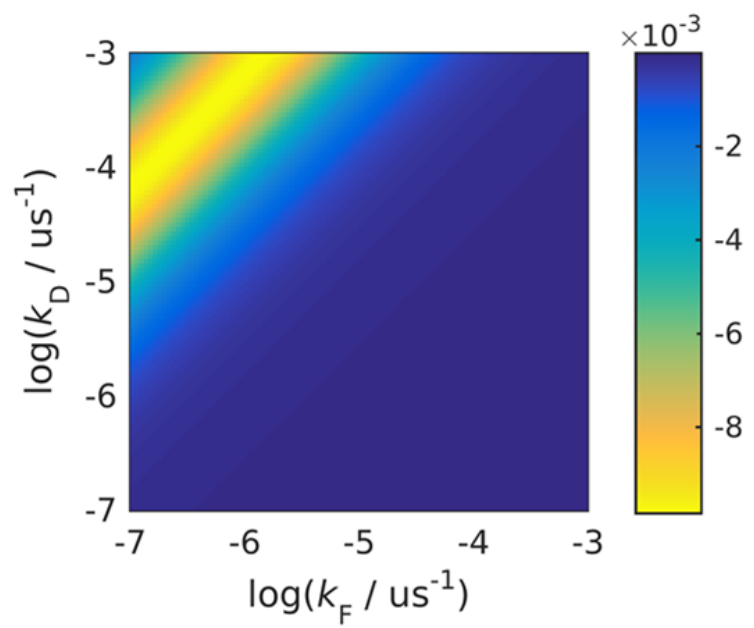

Supplementary Figure C8. Density plot of the relative changes in the total concentration of the flavin radical form of cryptochrome as a function of the radical termination rate constants $k_{\mathrm{F}}$ and $k_{\mathrm{D}}$. The values of the parameters are given Supplementary Table C2, except that $k_{\mathrm{EX}}$ has been reduced by a factor of 10 . 


\section{Bare-bones model of MFEs for inter- and intramolecular radical}

\section{pairs}

The kinetic model described above, while comprehensive, does not readily reveal the essential principles underlying the enhanced MFE. Here we present an approximate, pared down model that shows the same qualitative behaviour and affords a simple expression relating the amplification factor, $E$, to relevant rate constants.

\section{Intermolecular MFEs}

a)

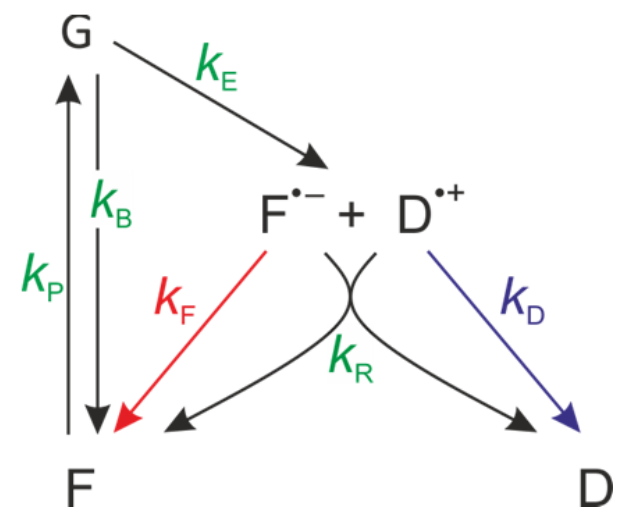

b)

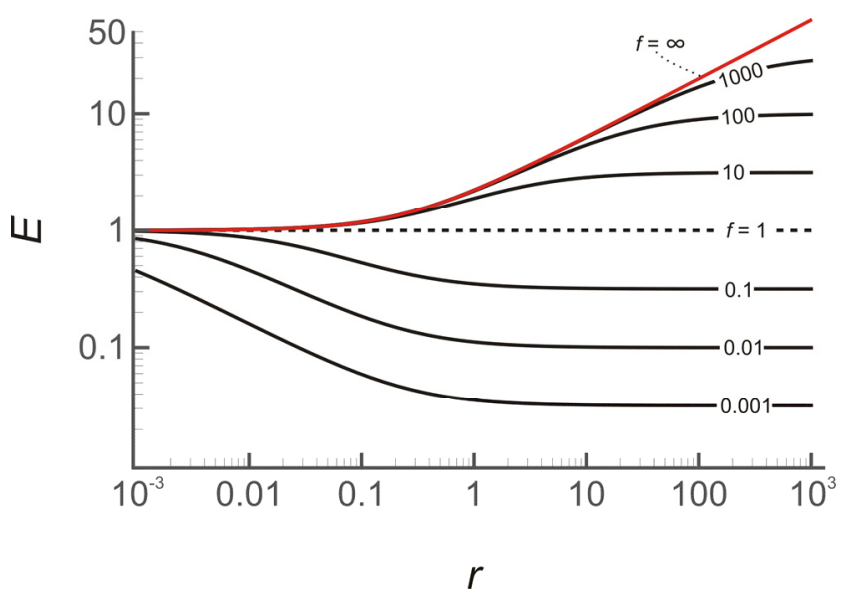

Supplementary Figure D1. a) Simplified reaction scheme for magnetic field effects on intermolecular flavin + donor reactions. The curved arrows indicate a bimolecular process. b) Dependence of the amplification factor $E$ on the parameter $r$ (defined in the text). The values of $f$ $=k_{\mathrm{D}} / k_{\mathrm{F}}$ are as indicated.

A simplified intermolecular radical pair reaction scheme is shown in Supplementary Fig. D1a. It comprises the diamagnetic, ground-state flavin $(F)$, uncorrelated free radicals $\left(F^{\circ-}\right.$ and $\left.D^{\circ+}\right)$, and a magnetically sensitive intermediate $(G)$, which accounts for the radical pair and all upstream, excited states. Magnetic field effects are introduced by allowing the rate constant with which $\mathrm{G}$ returns to $\mathrm{F}$, $k_{\mathrm{B}}\left(B_{0}\right)$, i.e. the recombination efficiency, to depend on the strength of the external magnetic field, $B_{0}$. The rate equations are as follows:

$$
\begin{aligned}
& \frac{\mathrm{d}}{\mathrm{d} t}[\mathrm{~F}]=-k_{\mathrm{P}}[\mathrm{F}]+k_{\mathrm{B}}\left(B_{0}\right)[\mathrm{G}]+k_{\mathrm{F}}\left[\mathrm{F}^{\cdot-}\right]+k_{\mathrm{R}}\left[\mathrm{F}^{\cdot-}\right]\left[\mathrm{D}^{\cdot+}\right] \\
& \frac{\mathrm{d}}{\mathrm{d} t}[\mathrm{G}]=-\left(k_{\mathrm{B}}\left(B_{0}\right)+k_{\mathrm{E}}\right)[\mathrm{G}]+k_{\mathrm{P}}[\mathrm{F}] \\
& \frac{\mathrm{d}}{\mathrm{d} t}\left[\mathrm{~F}^{\cdot-}\right]=k_{\mathrm{E}}[\mathrm{G}]-k_{\mathrm{F}}\left[\mathrm{F}^{\cdot-}\right]-k_{\mathrm{R}}\left[\mathrm{F}^{\cdot-}\right]\left[\mathrm{D}^{\cdot+}\right] \\
& \frac{\mathrm{d}}{\mathrm{d} t}\left[\mathrm{D}^{\cdot+}\right]=k_{\mathrm{E}}[\mathrm{G}]-k_{\mathrm{D}}\left[\mathrm{D}^{\cdot+}\right]-k_{\mathrm{R}}\left[\mathrm{F}^{\cdot-}\right]\left[\mathrm{D}^{\cdot+}\right] .
\end{aligned}
$$

The prompt MFE $\left(\chi_{\mathrm{p}}\right)$ is negligibly affected by the slow termination reactions (rate constants $k_{\mathrm{F}}$ and $k_{\mathrm{D}}$ ). Thus, to a good approximation, $\chi_{\mathrm{p}}$ can be evaluated from the steady state concentrations in the 
limit $k_{\mathrm{F}}=k_{\mathrm{D}}=0$. Using Equation (4) in combination with the requirement that the total concentration of flavin species is fixed,

$$
[\mathrm{F}]+\left[\mathrm{F}^{\cdot-}\right]=c_{0}
$$

we find:

$$
[\mathrm{F}]_{\mathrm{SS}}\left(k_{\mathrm{F}}=k_{\mathrm{D}}=0\right)=\frac{2 c_{0} k_{\mathrm{R}} k_{\mathrm{B}}+k_{\mathrm{E}}\left(k_{\mathrm{P}}+2 c_{0} k_{\mathrm{R}}\right)-\sqrt{k_{\mathrm{E}} k_{\mathrm{P}}\left(k_{\mathrm{E}} k_{\mathrm{P}}+4 c_{0} k_{\mathrm{R}}\left(k_{\mathrm{B}}+k_{\mathrm{E}}\right)\right)}}{2 k_{\mathrm{R}}\left(k_{\mathrm{B}}+k_{\mathrm{E}}\right)} .
$$

In the general case $\left(k_{\mathrm{F}} \neq 0\right.$ and $\left.k_{\mathrm{D}} \neq 0\right)$ in which the steady-state concentrations include the additional effects of the termination reactions, we obtain:

$[\mathrm{F}]_{\mathrm{SS}}=\frac{k_{\mathrm{D}} k_{\mathrm{E}} k_{P}+k_{\mathrm{F}}\left(k_{\mathrm{B}}+k_{\mathrm{E}}\right)\left(k_{\mathrm{D}}+2 c_{0} k_{\mathrm{R}}\right)-\sqrt{k_{\mathrm{D}}\left(k_{\mathrm{D}}\left(k_{\mathrm{F}}\left(k_{\mathrm{B}}+k_{\mathrm{E}}\right)+k_{\mathrm{E}} k_{\mathrm{P}}\right)^{2}+4 c_{0} k_{\mathrm{R}} k_{\mathrm{F}} k_{\mathrm{E}}\left(k_{\mathrm{B}}+k_{\mathrm{E}}\right) k_{\mathrm{P}}\right)}}{2 k_{\mathrm{F}}\left(k_{\mathrm{B}}+k_{\mathrm{E}}\right) k_{\mathrm{R}}}$.

The MFE is defined as the relative change in the steady-state concentration of $\mathrm{F}$ brought about by the action of the magnetic field. Since the MFEs, $\chi_{i}(i=d, p)$, are typically small, it is permissible to equate $\chi_{i}$ with a series expansion in $k_{\mathrm{B}}$ truncated after the linear term. Specifically:

$$
\chi_{i} \approx \frac{\mathrm{d} \ln [\mathrm{F}]_{\mathrm{SS}}}{\mathrm{d} k_{\mathrm{B}}} \Delta k_{\mathrm{B}}
$$

where $[\mathrm{F}]_{\mathrm{sS}}$ is given by Equations (6) and (7) for the prompt MFE, $\chi_{\mathrm{p}}$, and the delayed MFE, $\chi_{\mathrm{d}}$, respectively. $\Delta k_{\mathrm{B}}$ is the field-induced change in $k_{\mathrm{B}}$. Combining Equations (6), (7) and (8) gives:

$$
E=\frac{\chi_{\mathrm{e}}}{\chi_{\mathrm{p}}}=\frac{k_{\mathrm{D}} \sqrt{k_{\mathrm{E}} k_{P}\left(k_{\mathrm{E}} k_{\mathrm{P}}+4 c_{0} k_{\mathrm{R}}\left(k_{\mathrm{B}}+k_{\mathrm{E}}\right)\right)}}{\sqrt{k_{\mathrm{D}}\left(k_{\mathrm{D}}\left(k_{\mathrm{F}}\left(k_{\mathrm{B}}+k_{\mathrm{E}}\right)+k_{\mathrm{E}} k_{\mathrm{P}}\right)^{2}+4 c_{0} k_{\mathrm{R}} k_{\mathrm{F}} k_{\mathrm{E}} k_{\mathrm{P}}\left(k_{\mathrm{B}}+k_{\mathrm{E}}\right)\right)}} .
$$

Since $k_{\mathrm{D}}$ and $k_{\mathrm{F}}$ are assumed small compared to the other rate constants, Equation (9) can be expanded in powers of these rate constants, keeping their ratio constant, $f=k_{\mathrm{D}} / k_{\mathrm{F}}$, constant. Retaining only the first term, we obtain:

$$
E=\sqrt{\frac{1+4 r}{1+4 r / f}}
$$

where $r=\left(k_{\mathrm{R}} c_{0}\right) /\left(\phi_{\mathrm{E}} k_{\mathrm{P}}\right)$ and $\phi_{E}=k_{\mathrm{E}} /\left(k_{\mathrm{E}}+k_{\mathrm{B}}\right)$ denotes the quantum yield for the formation of free radicals. When $r \gg 1$ and $r \gg f$, Equation (10) implies that $E \approx \sqrt{f}$. Supplementary Fig. D1b illustrates the dependence of $E$ on $r$ for different ratios of $k_{\mathrm{D}}$ and $k_{\mathrm{F}}$. When $r$ is greater than about $100, E$ is indeed seen to be proportional to $\sqrt{f}$. 


\section{Intramolecular MFEs}

Amplified MFEs can also occur for radical pairs formed intramolecularly in a photocycle in which there are no bimolecular recombination reactions. A bare-bones reaction scheme that qualitatively accounts for MFEs in this case is shown in Supplementary Fig. D2a. Proceeding in a manner analogous to that for the intermolecular case, we find that the MFE enhancement for the groundstate $(F-D)$ is given by

$$
E=\frac{f^{2}+f+1}{\kappa f^{2}+f+\kappa}
$$

where $\kappa=k_{\mathrm{P}} /\left[k_{\mathrm{P}}+k_{\mathrm{B}}\left(B_{0}\right)\right]$. Note that when $k_{\mathrm{F}}=k_{\mathrm{D}}=0$, the MFE is $\chi_{\mathrm{p}}=\kappa \Delta k_{\mathrm{B}} / k_{\mathrm{B}}$. For an experiment that detects the fluorescence of the electron acceptor, $F$ (see Supplementary Fig. D2a), Equation (11) is applicable when the photoexcited state of $F$ is swiftly quenched in the state $F-D^{\circ+}$, e.g. by energy transfer. Note that $\kappa \leq 1$ so that an enhancement of the MFE $(E \geq 1)$ is expected irrespective of the value of $f=k_{\mathrm{D}} / k_{\mathrm{A}}$. This is strikingly different to the intermolecular case above.

If the populations of both $F-D^{\circ+}$ and $F-D$ are monitored via the fluorescence of $F$, the enhancement factor is given by:

$$
E=\frac{f^{2}+f+1}{\kappa f^{2}+f+\kappa}-\frac{1}{f(1-\kappa)+\kappa}
$$

and significant MFE enhancement results for $f>1$. If the fluorescence quantum yield is reduced by the presence of $D^{\circ+}$, Eqs. (12) and (11) provide lower and upper bounds on E. Supplementary Fig. D2b illustrates the dependence of $E$ on $f$ in these two limits.

a)

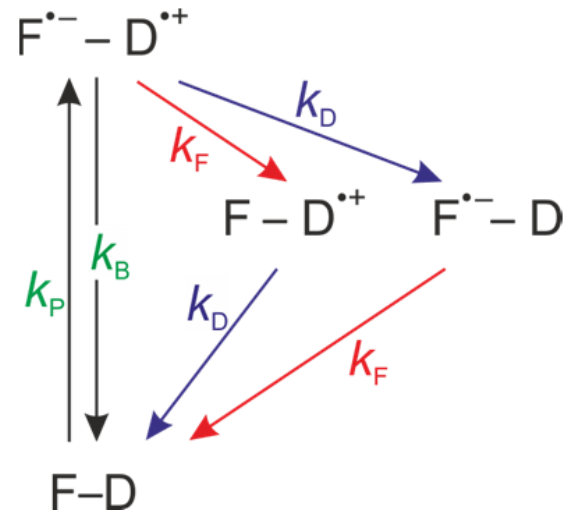

b)

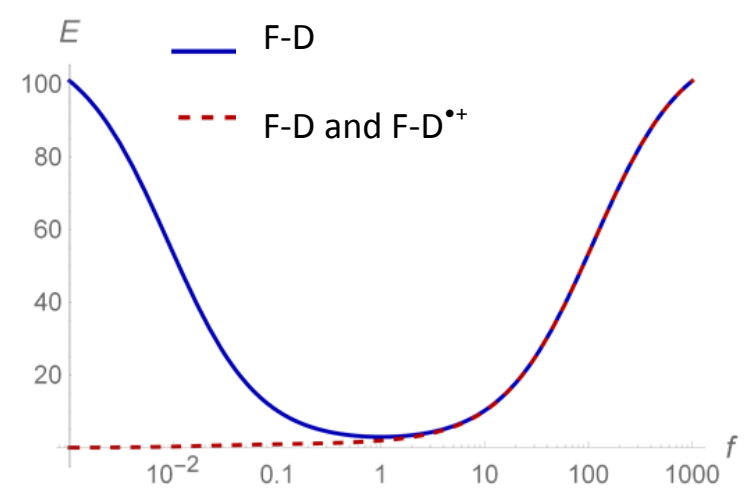

Supplementary Figure D2. a, Simplified reaction scheme for magnetic field effects on intramolecular flavin + donor reactions. $\mathbf{b}$, Dependence of the enhancement factor $E$ on $f=k_{\mathrm{D}} / k_{\mathrm{F}}$ for $\chi_{\mathrm{p}}=0.89 \%$. The blue line gives the MFE on the ground-state population. The dashed red line gives the MFE on the total concentration of $F$ irrespective of the oxidation state of the donor. The two calculations predict almost identical $E$ when $f>2$. 


\section{E. Flavin photochemistry}

\section{Transient absorption}

Transient absorption spectra for the FMN/lysozyme and FMN/Trp reactions were obtained using a Sirah Cobra Dye laser ( $450 \mathrm{~nm}, 3 \mathrm{~mJ}, 7 \mathrm{~ns}$ pulses) pumped by a Nd:YAG Continuum Surelite laser (355 $\mathrm{nm}, 100 \mathrm{~mJ}, 7 \mathrm{~ns}$ pulses). An Oriel xenon arc lamp ( $800 \mathrm{~W}$ ) provided the continuous UV-visible probe light. The probe light transmitted through the sample cell was passed to an Oriel monochromator to select a specific wavelength and then detected by a photo-multiplier tube. The time-resolved absorption profile, $\Delta A(t)$, was recorded as the difference in the absorption of the sample before and after photoexcitation, as a function of time after the pump pulse.

The transient absorption spectra for FMN/lysozyme and FMN/Trp (Supplementary Fig. E1) show that the flavin triplet excited state, ${ }^{3} \mathrm{FMN} *$, and the neutral flavin semiquinone radical, $\mathrm{FMNH}^{\circ}$, decay on the order of microseconds and seconds respectively, in agreement with prior studies. ${ }^{10,11}$ A SternVolmer plot for ${ }^{3} \mathrm{FMN}^{*}$ gave a ${ }^{3} \mathrm{FMN} *$-Trp quenching rate constant of $1.2 \times 10^{9} \mathrm{M}^{-1} \mathrm{~s}^{-1}$ close to the diffusion controlled limit.

a) $100 \mu \mathrm{M} F M N+0.5 \mathrm{mM}$ lysozyme

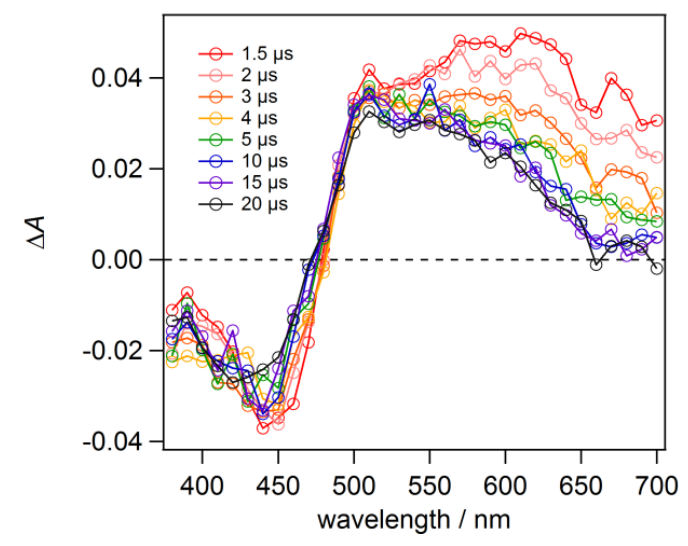

b) $200 \mu \mathrm{M} F M N+1.0 \mathrm{mM}$ Trp

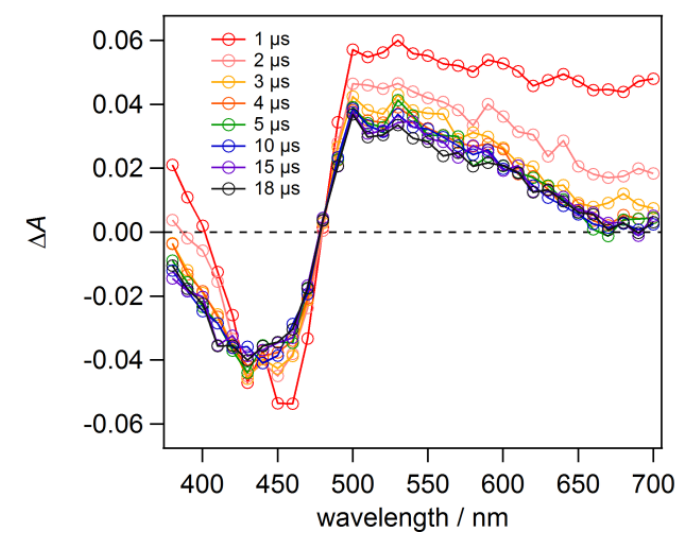

Supplementary Figure E1. Transient absorption spectra for a) FMN/lysozyme (triplet lifetime = $2.15 \pm 0.01 \mu \mathrm{s}$ ) and b) FMN/tryptophan (triplet lifetime $=0.62 \pm 0.01 \mu \mathrm{s}$ ) in aqueous solution.

\section{Reactivity of flavin radicals}

A significant reaction pathway for the neutral flavin semiquinone radical is the thermodynamically favourable disproportionation reaction to form the fully oxidized and fully reduced forms of the flavin:

$$
2 \mathrm{FH}^{\bullet} \rightarrow \mathrm{FH}_{2}+\mathrm{F}
$$

The disproportionation and comproportionation reactions having rate constants: $k_{\text {dis }}=5 \times 10^{8} \mathrm{M}^{-1} \mathrm{~s}^{-1}$ and $k_{\mathrm{com}}=1 \times 10^{6} \mathrm{M}^{-1} \mathrm{~s}^{-1}$, respectively. ${ }^{12,13}$ Oxidation of the fully reduced flavin by activated oxygen species such as hydrogen peroxide can recover the oxidized flavin:

$$
\mathrm{FH}_{2}+\mathrm{H}_{2} \mathrm{O}_{2} \rightarrow \mathrm{F}+2 \mathrm{H}_{2} \mathrm{O}
$$


Furthermore, there is a slow reaction pathway for the neutral semiquinone radical reacting with molecular oxygen ${ }^{12,13}$ with a rate constant $k_{\mathrm{O}_{2}} \approx 1 \times 10^{4} \mathrm{M}^{-1} \mathrm{~s}^{-1}$ :

$$
\mathrm{FH}^{\bullet}+\mathrm{O}_{2} \rightarrow \mathrm{F}+\mathrm{O}_{2}^{\bullet-}+\mathrm{H}^{+}
$$

However, oxidation of $\mathrm{FH}^{\bullet}$ radicals by the superoxide $\left.\mathrm{O}_{2}^{\bullet-}\right)$ formed in (15),

$$
\mathrm{FH}^{\bullet}+\mathrm{O}_{2}^{\bullet-}+\mathrm{H}^{+} \rightarrow \mathrm{F}+\mathrm{H}_{2} \mathrm{O}_{2}
$$

occurs much more rapidly $\left(k_{\mathrm{O}_{2}^{--}} \approx 1 \times 10^{8} \mathrm{M}^{-1} \mathrm{~s}^{-1}\right)$, meaning that the two reactions together are autocatalytic. $^{13}$

The disproportionation and molecular oxygen reactions are obvious candidates for MFE-enhancing termination processes in intermolecular photocycles. Both reactions proceed with a supra-linear dependence on the concentration of flavin radicals resulting in a reduction in $k_{\mathrm{D}} / k_{\mathrm{F}}$, and hence also in $E$, when either the flavin concentration or the pump power is increased (see above and main text).

Furthermore, the rate of reaction of flavin radicals with molecular oxygen is strongly dependent on their protonation state $\left(\mathrm{pK}_{\mathrm{a}}\left(\mathrm{FMNH}^{\circ}\right)=8.6\right)$ and therefore on the $\mathrm{pH}$. The equivalent reaction to (15) for $\mathrm{FMN}^{\bullet-}$ has a rate constant, $k_{\mathrm{O}_{2}} \approx 1 \times 10^{8} \mathrm{M}^{-1} \mathrm{~s}^{-1}$, that is four orders of magnitude faster than for $\mathrm{FMNH}^{\bullet}{ }^{12,13}$ This allows $E$ to be controlled by changing the $\mathrm{pH}$ of the solution.

To confirm the involvement of molecular oxygen, experiments were performed on de-oxygenated aqueous solutions of FMN $(10 \mu \mathrm{M})$ and tryptophan $(1.0 \mathrm{mM})$ prepared in a glove box under an argon atmosphere (Supplementary Fig. E2). Consistent with the above interpretation, we found an increased amplification factor $(E)$ in the absence of oxygen because of the reduced rate of removal of flavin radicals $\left(k_{\mathrm{F}}\right)$. The absolute amplitude of the prompt magnetic field effect is also slightly higher, probably due to a longer radical pair lifetime. De-oxygenation has an impact at several points in the photocycle (unlike, for example, adding TCEP whose only significant effect is to accelerate the reduction of the donor radical). In the absence of oxygen, flavin triplets accumulate leading to triplet-triplet annihilation $\left(\mathrm{T}_{1}+\mathrm{T}_{1} \rightarrow \mathrm{S}_{1}+\mathrm{S}_{0}\right)$ and delayed fluorescence $\left(\mathrm{S}_{1} \rightarrow \mathrm{S}_{0}+h v_{\mathrm{F}}\right)$ which complicates matters considerably. Additionally, in the absence of oxygen, the flavin radicals are very long lived meaning that there is a much greater depletion of the ground state reactants and consequently weak fluorescence from the desired route $\left(\mathrm{S}_{0}+h v \rightarrow \mathrm{S}_{1} ; \mathrm{S}_{1} \rightarrow \mathrm{S}_{0}+h \nu_{\mathrm{F}}\right)$. In short, the presence of oxygen is desirable in the sense that it "closes" the photocycle and gives favourable cycle times. 


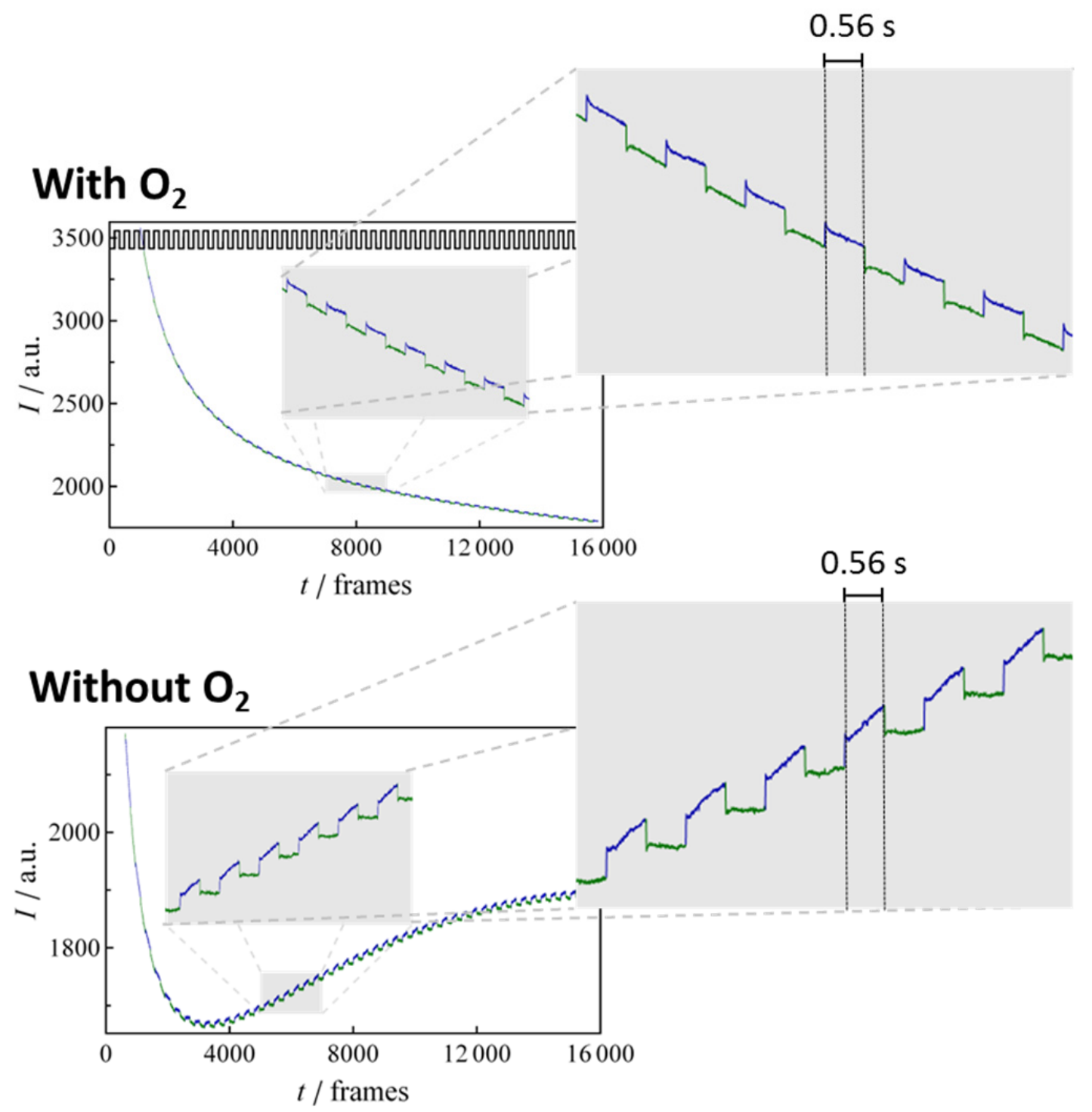

Supplementary Figure E2. Fluorescence intensities of aqueous solutions of FMN (10 $\mu \mathrm{M})$ and tryptophan $(1.0 \mathrm{mM})$ recorded under oxygenated and de-oxygenated conditions. 


\section{F. Switched light intensity}

Corroboration of our interpretation of the MFE amplification effect was obtained from experiments in which the light intensity was periodically adjusted (in the absence of a magnetic field) as an alternative way of shifting the position of the photo-stationary state (Supplementary Fig. F1a). Attenuation of the light intensity was achieved by square-wave, on-off modulation of the $350 \mathrm{~mW}$ laser output varying the duty cycle to obtain the reduced intensities $(80 \%, 70 \%, 60 \%, 50 \%$ and $20 \%)$ shown in the figure. When the light intensity was abruptly reduced, a prompt fluorescence component was observed followed by a slow delayed component. This is reproduced by simulations (Supplementary Fig. F1b) with the same parameter values used previously (Supplementary Table C1).

(a)

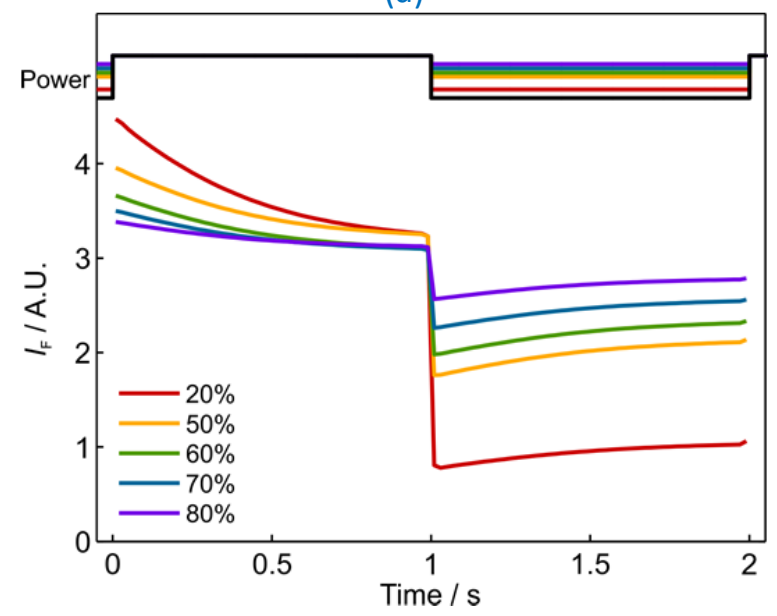

(b)

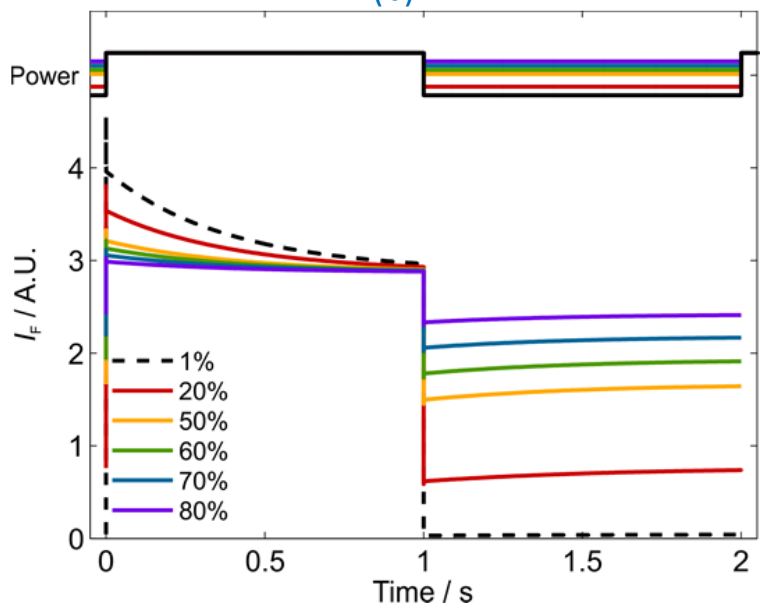

Supplementary Figure F1. (a) Fluorescence intensities of an aqueous solution of FMN $(10 \mu \mathrm{M})$ and lysozyme $(0.5 \mathrm{mM})$ recorded using a $405 \mathrm{~nm}$ diode laser. The laser power $(350 \mathrm{~mW})$ was periodically attenuated by $20-80 \%$ after $1.0 \mathrm{~s}$ of irradiation and returned to $350 \mathrm{~mW}$ after a further $1.0 \mathrm{~s}$. (b) Simulation of this experiment using $k_{\mathrm{F}}=1 \mathrm{~s}^{-1}, k_{\mathrm{D}}=10.0 \mathrm{~s}^{-1}, k_{\mathrm{EX}}=1.73 \times 10^{3} \mathrm{~s}^{-1}$ and other parameter values as in Supplementary Table $\mathrm{C1}$. 


\section{Supplementary References}

1. Evans E. W., et al. Sensitive fluorescence-based detection of magnetic field effects in photoreactions of flavins. Phys. Chem. Chem. Phys. 17, 18456-18463 (2015).

2. Courson D. S. \& Rock R. S. Fast benchtop fabrication of laminar flow chambers for advanced microscopy techniques. Plos One 4, e6479 (2009).

3. Dodson C. A., et al. Fluorescence-detected magnetic field effects on radical pair reactions from femtolitre volumes. Chem. Commun. 51, 8023-8026 (2015).

4. $\quad$ Press W. H., Teukolsky S. A., Vetterling W. T. \& Flannery B. P. Numerical Recipes 3rd edition: The Art of Scientific Computing. Cambridge University Press: Cambridge, 2007.

5. Holzer W., et al. Photo-induced degradation of some flavins in aqueous solution. Chem. Phys. 308, 69-78 (2005).

6. Murakami M., Maeda K. \& Arai T. Dynamics of intramolecular electron transfer reaction of FAD studied by magnetic field effects on transient absorption spectra. J. Phys. Chem. A 109, 5793-5800 (2005).

7. Losi A., Polverini E., Quest B. \& Gartner W. First evidence for phototropin-related blue-light receptors in prokaryotes. Biophys. J. 82, 2627-2634 (2002).

8. Holzer W., Penzkofer A., Fuhrmann M. \& Hegemann P. Spectroscopic characterization of flavin mononucleotide bound to the LOV1 domain of Phot1 from Chlamydomonas reinhardtii. Photochem. Photobiol. 75, 479-487 (2002).

9. Maeda K., et al. Magnetically sensitive light-induced reactions in cryptochrome are consistent with its proposed role as a magnetoreceptor. Proc. Natl. Acad. Sci. USA 109, 47744779 (2012).

10. Miura T., Maeda K. \& Arai T. Effect of coulomb interaction on the dynamics of the radical pair in the system of flavin mononucleotide and hen egg-white lysozyme (HEWL) studied by a magnetic field effect. J. Phys. Chem. B 107, 6474-6478 (2003).

11. Tsentalovich Y. P., Lopez J. J., Hore P. J. \& Sagdeev R. Z. Mechanisms of reactions of flavin mononucleotide triplet with aromatic amino acids. Spectrochim Acta A 58, 2043-2050 (2002).

12. Massey V. Activation of molecular-oxygen by flavins and flavoproteins. J. Biol. Chem. 269, 22459-22462 (1994).

13. Vaish S. P. \& Tollin G. Flash photolysis of flavins. V. Oxidation and disproportionation of flavin radicals. J. Bioenerg. 2, 61-72 (1971). 


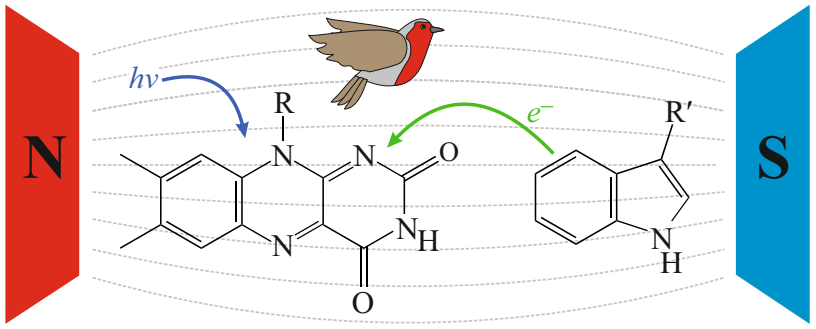




\section{Summary for Table of Contents}

Proposed as the source of the light-dependent magnetic compass in migratory birds, the radical pair mechanism is thought to operate in flavoproteins in the retina. We demonstrate that the primary magnetic field effect on flavin photoreactions can be chemically amplified by slow radical termination reactions under conditions of continuous photoexcitation. 


\section{Response to Editor}

We have implemented all the changes you requested:

- $\quad$ Fig. 3 has been reformatted.

- $B_{0}$ in Fig. 2 has been defined.

- The main points of interest in Figs 3, 4 and 6 have been outlined briefly.

- A Graphical Abstract has been provided.

- A Table of Contents summary has been provided.

- The Supplementary Information now has an index.

- All Figures and Tables in the Supplementary Information are now designated Supplementary Figures and Supplementary Tables.

- Each section of the Supplementary Information is now referred to explicitly in the main text. 